\title{
Targeting Histone Deacetylase-3 Blocked Epithelial- Mesenchymal Plasticity And Metastatic Dissemination In Gastric Cancer
}

\section{Sheng-Mao Wu}

NCHU: National Chung Hsing University

Yee-Jee Jan

Taichung VGH: Taichung Veterans General Hospital

Shih-Chuan Tsai

Taichung VGH: Taichung Veterans General Hospital

Hung-Chuan Pan

Taichung Veterans General Hospital

Chin-Chang Shen

Institute of Nuclear Energy Research

Cheng-Ning Yang

NTU: National Taiwan University

Shu-Hua Lee

NCHU: National Chung Hsing University

Shing-Hwa Liu

NTU: National Taiwan University

Chien-Shan Chiu

Taichung Veterans General Hospital

Jack L. Arbiser

Emory University School of Medicine

Menghsiao Meng

NCHU: National Chung Hsing University

Meei-Ling Sheu ( $\triangle$ mlsheu@nchu.edu.tw)

NCHU: National Chung Hsing University https://orcid.org/0000-0002-0431-1282

\section{Research Article}

Keywords: Histone deacetylase inhibitors, Histone deacetylase 3, peritoneal dissemination, epithelialmesenchymal transition, endoplasmic reticulum stress

Posted Date: June 14th, 2021 
DOl: https://doi.org/10.21203/rs.3.rs-552157/v1

License: (c) (1) This work is licensed under a Creative Commons Attribution 4.0 International License. Read Full License

Version of Record: A version of this preprint was published at Cell Biology and Toxicology on January 1st, 2022. See the published version at https://doi.org/10.1007/s10565-021-09673-2. 


\section{Abstract}

Background and purpose: Histone deacetylase (HDAC) inhibitors (HDIs) were shown to modulate the epithelial-mesenchymal transition (EMT) progression and inhibit the migration and invasion of cancer cells. Emerging as a novel class of anti-cancer drugs, HDIs have attracted much attention in the field of drug discovery. This study aimed to the underlying mechanisms of Honokiol in preventing the metastatic dissemination of gastric cancer cells by inhibiting HDAC3 activity/expression.

Experimental Approach: Clinical pathological analysis was performed to determine the relationship between HDAC3 and tumor progression. The effects of Honokiol on pharmacological characterization, functional, transcriptional activities, organelle structure changes, and molecular signalings were analyzed using binding assays, Differential Scanning Calorimetry, luciferase reporter assay, HDAC3 activity, ER stress response element activity, transmission electron microscopy, immune-blotting and Wnt/b-catenin activity assays. The in vivo effects of Honokiol on peritoneal dissemination were determined by a mouse model and detected by the PET/CT tomography.

Key Results: HDAC3 over-expression was correlated with poor prognosis. Honokiol significantly abolished HDAC3 activity (Y298) via inhibition of NFKBp65/CEBPb signaling, which was reversed by the overexpression of plasmids of NFKBp65/CEBPb. Honokiol-inhibited NFKBp65/CEBPb activation could be reversed by 4-phenylbutyric acid (a chemical chaperone) and calpain-2 gene silencing. Honokiol increased ER stress markers and inhibited EMT-associated epithelial markers, but decreased Wnt/ $\beta$ catenin activity. Suppression of HDAC3 by both Honokiol and HDAC3 gene silencing decreased cell migration and invasion in vitro and metastasis in vivo.

Conclusions and Implications: Honokiol acts by suppressing HDAC3-mediated EMT and metastatic signaling. By prohibiting $H D A C 3$, metastatic dissemination of gastric cancer may be blocked.

\section{What Is Already Known}

- Honokiol suppresses cancer cell growth through its anti-epithelial mesenchymal transition (EMT) and anti-peritoneal metastatic effects.

- Targeting endoplasmic reticulum (ER) stress can restrain tumorigenesis and inhibit EMT in the metastatic progression of tumors.

\section{What this study adds}

- Honokiol significantly abolished HDAC3 activity on catalytic tyrosine 298 residue site.

- Honokiol-induced ER stress markedly inhibited HDAC3 expression via inhibition of NFKBp65/CEBPb signaling.

\section{What is the clinical significance}


- HDAC3, which is a positive regulator of metastatic gastric cancer cell growth, can be significantly inhibited by Honokiol.

- Opportunities for HDAC3 inhibition may be a potential therapeutic target for preventing gastric cancer metastatic dissemination.

\section{Introduction}

The incidence and mortality of cancers, including gastric cancer, is linked with tumor progression, from localized primary tumors to more advanced stages that metastasize and involve multiple organs (Brabletz, 2012; Gherardi et al., 2012; Williams et al., 2019). In cancers of the gastrointestinal tract, the peritoneum is the primary site of metastasis. The spread is associated with the loss of epithelial characteristics and the acquisition of a mesenchymal phenotype, which initiates invasion and metastasis (Brabletz et al., 2018). Gastric cancer is the second leading cause of cancer-related deaths globally. Most patients will relapse or have local recurrence after definitive surgical treatment. Moreover, patients with gastric cancer have a high risk of lymph node metastases and poor prognosis due to extensive invasion (Juttner et al., 2006; Maehara et al., 1999). The major cause of death is the generation of distant metastasis, which places substantial limits to successful therapeutic interventions (Liu et al., 2015; Wu et al., 2016b). The overall five-year survival rate of patients with advanced gastric cancer even after treatment remains unsatisfactory, without significant improvement in the past decades (Liu et al., 2015; Wu et al., 2016b). Thus, there is an urgent need to investigate the molecular mechanisms of gastric cancer metastasis and identify the novel biomarkers that may predict metastasis.

Recent evidence has shown a close relationship between epithelial-to-mesenchymal transition (EMT) and disease progression (Chiu et al., 2019; Lai et al., 2014; Liu et al., 2015; Wu et al., 2016b). The EMT is considered a key process in the metastatic step that confers fundamental abilities of tumor cells migration, invasiveness, and anoikis resistance, during which non-motile, polarized epithelial cells dissolve their cell-to-cell junctions and are convert into individual, motile mesenchymal cells (Brabletz et al., 2018; Gherardi et al., 2012). Reduction or loss of cell surface E-cadherin is a classic EMT hallmark, while E-cadherin down-regulation has been correlated with short disease-free survival and poor outcomes (Brabletz et al., 2018; Chiu et al., 2019; Wu et al., 2016b).

During tumor metastasis, EMT may give rise to the dissemination of single tumor cells from the primary epithelial tumor. These are disrupted by numerous signaling pathways and regulatory transcriptional networks, and controlled by distinct signaling molecules in the tumor micro-environment (Jung et al., 2015; Tsai and Yang, 2013). As it can be regulated by transcription factors, EMT progression requires robust transcriptional machinery and post-transcriptional mechanisms, which consist largely of developmental transcription factors that regulate and coordinate epithelial and mesenchymal markers (Brabletz et al., 2018; Chiu et al., 2019; Lai et al., 2014; Liu et al., 2015; Wu et al., 2016b). Dissecting the molecular mechanisms that regulate EMT and E-cadherin expression are crucial in understanding tumor invasiveness and metastasis. 
Discovered in 2000, Class I histone deacetylase (HDAC3) is the predominant HDAC associated with nuclear receptor co-repressor 1 (NCoR1 or NCoR) and the silencing mediator of retinoic acid and thyroid hormone receptor (SMRT, also known as NCoR2) (Mani and Barton, 2011). A previous study has demonstrated that hypoxia-induced HIF-1a-mediated activation of HDAC3 occurs in both epithelial and mesenchymal cells and is essential for EMT and metastasis (Wu et al., 2011). In epithelial cells, hypoxia induces HDAC3 expression and its enzymatic activity towards the removal of the acetyl group of histone 3 lysine 4 (H3K4Ac). This facilitates epithelial gene repression, the activation of mesenchymal gene expression, and the repression of the epithelial-associated gene chromatin structure (Mani and Barton, 2011). One crucial mechanisms is via the interplay between HDAC3 and WDR5 to regulate EMT marker genes such as E-cadherin and vimentin (Wu et al., 2012; Wu et al., 2011). Gene silencing of HDAC3 abolishes the EMT marker gene regulation in response to hypoxia-induced condition (Chen et al., 2016). Thus, HDAC3 plays a decisive role in hypoxia-induced EMT. However, the role of HDACs in EMT is controversial. Several studies reported that HDACs inhibit EMT but this is not always the case. HDAC inhibitors may reinforce EMT progression in various conditions (Debeb et al., 2012; Ji et al., 2015; Kong et al., 2012; Sakamoto et al., 2016; Wawruszak et al., 2019a; Wawruszak et al., 2019b; Wu et al., 2016a).

Moreover, all mammalian HDACs contain putative sites of post-translational modification, including phosphorylation sites that may alter HDAC3 activity and stability, or protein complex assembly similar to how many nuclear receptor co-regulators work (Emmett and Lazar, 2019). Distinct mechanisms also show Y298F and K25A mutations that abolish the HDAC3 enzymatic activity (Sun et al., 2013). The crystal structure of a eukaryotic zinc-dependent histone deacetylase reveals highly conserved Tyr residues (Y298 in HDAC3) located within the active site. These are catalytically essential in stabilizing the tetrahedral intermediate and in polarizing the substrate carbonyl for nucleophilic attack in coordination with the $\mathrm{Zn}$ ion. The mutation of Y298F (YF) causes the complete inactivation of in vitro-translated HDAC3 proteins. More importantly, their interactions with a number of miRNAs or transcription factors may block the HDAC3-regulated EMT and metastasis by repressing HDAC3 expression (Ma et al., 2020). This presents a regulatory network that involves many key players in the EMT program.

Honokiol is a small-molecule polyphenol attained from the bark of the genus Magnolia. It has potential anti-tumor therapy in various cancer models (Fried and Arbiser, 2009). Intravenous injection of 5-10 $\mathrm{mg} / \mathrm{kg}$ Honokiol in experimental rats reveal half-lives of $49.22 \pm 6.68$ and $56.24 \pm 7.30 \mathrm{~min}$ (Chiu et al., 2019; Fried and Arbiser, 2009). Meanwhile, results of intra-peritoneal injection following $250 \mathrm{mg} / \mathrm{kg}$ can yield a $t_{1 / 2}$ of 4-6 h, with a $t_{\max }$ of about $20-30$ min in mice. Honokiol retention time is 4.94 min (Liu et al., 2015). Honokiol has unusual structural motifs among phenolic antioxidants and these motifs feature isomeric bisphenolic cores bearing allyl substituents (Amorati et al., 2015). Our previous serial study has revealed that Honokiol-induces cancer cell apoptosis and inhibits tumorigenesis and metastasis of gastric cancer cells via endoplasmic reticulum (ER) stress pathways and specific targeting of key calpain activation, as well as the down-regulation of CRT, PPARy, Grp94, COX-2, STAT-3, and Tpl-2 proteins. However, the anti-EMT potential of Honokiol and its correlation with HDAC3 regulation have not been investigated in a gastric cancer model. 
The present study aimed to clarify the role of Honokiol in suppressing HDAC3 activation on EMT and metastasis in gastric cancer. This is the first study to elucidate the role of Honokiol-induced changes in HDAC3 (Y298) site and C/EBPß, NFKB, on EMT and on peritoneal dissemination in gastric cancer. The results reveal that the invasiveness of gastric cancer cells, largely dependent on the over-expression of the $\mathrm{C} / \mathrm{EBP} / \mathrm{NFKB} / \mathrm{HDAC} 3$ signaling cascade, is indispensable for the invasiveness of pathologic tissues. Such findings provide extremely valuable information regarding the target genes in extracted clinical tissue that may be used for survival analysis. The clinical tissues reveal HDAC3 over-expression that is associated with tumor recurrence and poor prognosis. Further, through in vivo and in vitro studies that tracked gastric cancer cells in a mouse model of peritoneal carcinomatosis, this study demonstrates for the first time how Honokiol therapy, pharmacological inhibition, and gene silencing of HDAC3 effectively suppress the parietal peritoneum, thereby eliminating metastatic cancer cells. The HDAC3 knockdown in cancer cells inhibits EMT markers and activates endoplasmic reticulum (ER) stress in vivo.

\section{Material And Methods}

Most of the methods used here have been published previously but are repeated here for clarity (Chiu et al., 2019; Lee et al., 2013; Liu et al., 2015; Liu et al., 2010; Liu et al., 2012; Pan et al., 2013a; Pan et al., 2013b; Wu et al., 2016b). Honokiol was obtained from the Wako Chemical Company (Osaka, Japan).

\section{Cell culture}

Cell culture systems were performed as previously described (Wu et al., 2016b). The cell bank of Taipei Veterans General Hospital (Taiwan) supplied the human gastric cancer cell lines, AGS (moderately differentiated gastric adenocarcinoma) and MKN 45 or SCM-1 cells (poorly differentiated gastric adenocarcinoma). Cells were grown in RPMI medium supplemented with $10 \% \mathrm{FBS}, 100 \mathrm{U} / \mathrm{ml}$ penicillin, and $100 \mathrm{mg} / \mathrm{ml}$ streptomycin (complete medium) at $37^{\circ} \mathrm{C}$ in humidified incubator with $5 \% \mathrm{CO}_{2}$. During experiments, cells were plated in six-well plates cultured with serum-free medium (starved medium) overnight, and then treated with drugs (honokiol or melatonin) with various concentrations for various time intervals. In some experiments, transfection of cancer cells was performed using a Lipofectin reagent (Invitrogen) according to the manufacturer's instructions. The efficiency of transfection ( 90\%) was determined using an equal amount of a plasmid that encoded the green fluorescent protein under the cytomegalovirus promoter.

\section{Tissue samples and clinical data collection}

This study analyzed 40 patients with gastric cancer. The institutional review board of Taichung Veterans General Hospital approved the study protocol (Approval No CE 17096B). Data obtained from the registry of the Cancer Institute of Tissue Bank included age, sex, differentiation, tumor location, tumor size, depth of tumor invasion ( $\mathrm{T}$ stage) and number of metastatic lymph nodes ( $\mathrm{N}$ stage) (both based on the seventh edition UICC TNM classification for gastric cancer), extent of lymph node metastasis, and Lauren classification. Correlations between HDAC3 expression and the clinico-pathologic characteristics of 
patients were summarized. Fisher's exact test was used for the histologic analysis of tumor stage, tumor grade, and distant metastasis, while one-way analysis of variance (ANOVA) was used to compare clinicopathologic characteristics. Statistical significance was set at $p<0.05$.

\section{Ligand Binding assays}

Study were conducted as previously described in $10 \mathrm{mM}$ 2-(N-morpholino)ethanesulfonic acid (MES) buffer containing10 mM MnCl2, $1 \mathrm{mM}$ EDTA, adjusted to $\mathrm{pH} 6.0$ with $\mathrm{KOH}$ (Davis and Sharif, 2000). Initial tissue linearity studies were carried out using $0.1 \pm 200 \mu \mathrm{g}$ of cell containing the recombinant human HDAC3 per $0.5 \mathrm{ml}$ total volume in 96-well deep well assay blocks (Matrix Technologies Corp., Hudson, $\mathrm{NH}$, U.S.A.), using 300 pM [3H]-Honokiol (PerkinElmer Inc.). Protein were thawed quickly, diluted to the desired concentration in the binding buffer, and mixed to a homogeneous suspension prior to dispensation. After addition of the radioligand, the assay mixtures were incubated for $120 \mathrm{~min}$ on a rotary shaker (50 r.p.m.). The study was terminated by rapid vacuum filtration on Whatman GF/B glass filter mats (previously soaked in $0.5 \%$ polyetheleneimine) using cold MES buffer. Bound radioligand was then quantitated by liquid scintillation counting. (Packard BioScience).

\section{Differential scanning calorimetry (DSC)}

The methodology and rationale for DSC analysis (PerkinElmer DSC 7) are summarized as described previously and references contained within(Martin et al., 2013). Although some HDAC3 molecules may have nucleotide bound at the end of the purification, this will be released from the protein before the protein unfolding.

\section{Immuno-histochemistry}

In the human gastric cancer tissues, $5 \mu$ m thick sections were cut from $10 \%$ formalin-fixed samples and stained for specific antibodies using anti-primary antibodies. Immuno-histochemistry (IHC) analysis was performed as described previously (Liu et al., 2015). The antibodies used were listed and described in supplementary Tables 1 and 2.

\section{HDAC3 activity}

A HDAC Activity Assay Kit was used to examine HDAC activity, following the manufacturer's protocol (Abnova Corporation; Catalog Number KA3731). The assay kit provided a positive control (a HeLa nuclear extract), a deacetylated HDAC assay standard, and a control inhibitor (trichostatin A; TSA), as well as a colorimetric HDAC substrate [R-H-K-K(Ac)-AFC] to release the AFC molecule, which could be fluorometrically detected $(\mathrm{Ex} / \mathrm{Em}=380 / 500 \mathrm{~nm})$.

\section{Luciferase reporter assay}

Genetic reporters are used as indicators gene expression studies with accompanying cellular events (Pan et al., 2013a). Cells at $60 \%$ confluence were co-transfected with $0.2 \mu \mathrm{g}$ of the promoter reporter construct 
CCAAT/enhancer-binding protein B (C/EBP 3$), N F K B$ activator, and $0.05 \mu \mathrm{g}$ of a thymidine kinase promoter driven Renilla luciferase vector (pRLTK; Promega, Mannheim, Germany). The pRL-tk-LUC vector coding for a Renilla luciferase under control of a constitutively active thymidine kinase promoter was co-transfected to correct for transfection efficiency. After incubation, the cells were lysed and processed using the Dual Luciferase Kit (Promega) as described by the manufacturer. Luciferase activity was normalized against the Renilla firefly activity for transfection efficiency and recorded by a luminometer (LKB, Rockville, MD, USA). Experiments were performed in triplicate, unless stated otherwise.

\section{Endoplasmic reticulum stress response element (ERSE)}

Endoplasmic reticulum stress measurements were performed using the Cignal ERSE Reporter Luciferase assay kit (SA Biosciences, QIAGEN, Frederick, MD, USA). The ERSE reporter assay was a mixture of an ERSE-responsive luciferase construct and a constitutively expressed Renilla luciferase construct. The diluted transfection provided ready reporter, negative control, positive control formulations, and relevant nucleic acid tests. Overnight post-transfection, the transfected cells were treated with Honokiol and ER stress activator. Activities of the signaling pathways were investigated using dual luciferase assay.

\section{Endoplasmic reticulum stress response element (ERSE)}

TEM was performed as previously described (Pan et al., 2013b). Cells were treated with or without Honokiol or shHDAC3 for 18 hours and then harvested and fixed with $4 \%$ glutaraldehyde and $2.5 \%$ paraformaldehyde dissolved in $0.1 \mathrm{M}$ sodium cacodylate. The cells were then post-fixed in $1 \%$ osmium tetraoxide, dehydrated in ethanol, and embedded in araldite. Sections on grids were counter-stained with uranyl acetate and lead citrate, and examined in a JEM 1200 EX TEM (JEOL, Peabody, MA, USA) at an accelerating voltage of $80 \mathrm{kV}$.

\section{Transfection}

Cancer cells AGS, SCM1, and MKN45 were transfected with shRNA (National RNAi Core Facility Platform, Taipei, Taiwan) or over-expressed plasmid $1 \mu \mathrm{g} / \mathrm{ml}$ pcDNA (Genome Research Center, National Yang-Ming University) using a Lipofectin reagent (Invitrogen), in accordance with the manufacturer's instructions.

\section{Immuno-blotting}

The preparation of whole-cell lysates of gastric cancer cells for immuno-blotting was performed as previously described (e.g. Liu et al., 2015; Pan et al., 2013a). The antibodies used in the present study were also listed in supplementary Tables 1 and 2. Detection was performed by ECL (Amersham) and by chemiluminescence using Kodak X-Omat film. The immunoblot assay was independently repeated five times.

\section{Wound-healing cell migration assay and Matrigel invasion assay}


After transfection, the cells were seeded at $3.5 \times 10^{5}$ cells/well in 12-well plates. At $100 \%$ confluence, the cells were scraped by a sterile tip of a $1000 \mu$ pipette to generate an artificial "wound". Two parallel wounds were created using a plastic pipette tip. The cells were further grown in a culture medium with $2 \%$ FBS. The images were collected at 0 and $18 \mathrm{~h}$ using a microscope. Migration capacity was quantified by measuring the change of "wound" width. This assay was independently repeated five times.

For the Matrigel invasion assay, $3 \times 10^{5}$ cells/well were seeded in the upper chamber that was coated with Matrigel (BD Bioscience, San Diego, CA, USA). After $48 \mathrm{~h}$ at $37^{\circ} \mathrm{C}$ and $5 \% \mathrm{CO}_{2}$, the cells present on the lower surface of the insert were stained with Diff-Quik stain (Biochemical Sciences, Inc., Swedesboro, NJ, USA). Cells that invaded through the Matrigel-coated membrane were microscopically enumerated. Cell staining from three randomly selected fields were photographed using a CKX41 inverted microscope (Olympus Corp). The mean value was recorded. All experiments were performed in triplicate; each being repeated at least five times. And this variability of the mean values is represented by the SD.

\section{Experimental animals}

All of animal design and models are used have approval and all procedures has been conform to the guidelines from the NIH Guide for the Care and Use of Laboratory Animals. All animal studies were approved by the appropriate institutional ethical committee of the Taichung Veterans General Hospital

Taiwan (Approval No La-1061488). For euthanasia, mice during continuous deep were anaesthetized with isoflurane inhalation (induction: $3 \%$, maintenance: $1-2 \%)$ in medical air $(0.4 \mathrm{~L} / \mathrm{min})$. The animals were sacrificed by cervical dislocation under isoflurane.

\section{Xenograft tumor mouse model and positron emission tomography-computed tomography (PET/CT)}

Cell culture systems were used as described previously (Chiu et al., 2019). Imaging studies were performed using positron emission tomography-computed tomography (PET/CT) also as previously described. To evaluate for peritoneal metastasis, MKN45 cells were inoculated into the peritoneal cavity of BALB/c nude mice. Peritoneal tumors in the nude mice were established by PET/CT surveillance 5-7 days after the injection. The mice were given intra-peritoneal injections of Honokiol ( $5 \mathrm{mg} / \mathrm{kg} / \mathrm{twice}$ per week) for 28 days.

Changes in Honokiol on the peritoneal dissemination were evaluated by PET/CT. The mice were sacrificed under anesthesia and examined macroscopically for the presence of peritoneal metastasis. The tumors were excised, cut into blocks, fixed in $10 \%$ formalin, and embedded in paraffin blocks or snapfrozen in liquid nitrogen. All images were analyzed and interpreted by senior nuclear medicine physicians with all available clinical information. Correlative conventional imaging was used for anatomic guidance.

\section{TOP/FOP luciferase reporter assay}

To assess the transcriptional activity of $\beta$-catenin in gastric cancer cells, the TOP/FOP reporter system using the dualluciferase kit (Dual-GloTM Luciferase Assay System, Promega, Madison, WI, USA) were 
used. Cells were transiently transfected with $1 \mu \mathrm{g}$ of constitutively active vector encoding thymidine kinase promoter-Renilla luciferase reporter plasmid (pRL-TK) (Promega) and $\beta$-catenin responsive firefly luciferase reporter plasmid TopFlash (Millipore, Billerica, MA, USA), or the negative control FopFlash (Millipore) using lipofectin. Cells were harvested after $24 \mathrm{hrs}$ in culture and both firefly and Renilla luciferase activities were measured in duplicate/triplicate according to the manufacturer's instructions. The firefly luciferase activity was normalized against the Renilla luciferase activity and the fold increase in TOPFlash activity compared to FOPFlash was reported.

To assess the function of Honokiol on $\beta$-catenin transcriptional activity, cells were co-transfected with TopFlash or FopFlash and small hairpin RNA (shRNA) using lipofectin. The cells were harvested after 36 hrs for luciferase measurements. Luminescence was read using the Sirius luminometer (Berthold Detection System, Pforzheim, Germany).

\section{Statistical analyses}

Values were presented as mean \pm SD. Analysis of variance (ANOVA), followed by Fisher's least significant difference test, was performed for all data. Statistical significance was set at $p<0.05$.

\section{Results}

\section{High HDAC3 expressions decreased overall survival probability in gastric cancer patients}

Data from the Genotype-Tissue Expression project and the Cancer Genome Atlas were first integrated to comprehensively analyze the transcriptomes of 172 healthy and 414 tumor tissues (Fig. 1A). By webbased correlation, there was a markedly positive correlation between the high expressions of HDAC3 in 876 patients with gastric cancer, using the selected parameters and run on by Kaplan-Meier plotter (KMplot.com), Probability GSE216326 dataset, or by TCGA (Fig. 1B). The desired Affymetrix IDs is valid: 216326_at (HDAC3). Survival curves were plotted for all patients with gastric cancer $(n=876)$ using the Kaplan-Meier analysis, with HR of $1.42(1.19-1.68)$ and logrank $P=5.5 e-5$. In addition, similar patterns were also found in SurvExpress Web resource in stomach cancer of the TCGA dataset $(n=57)$ (Fig. 1C).

Immuno-histochemical analysis of human gastric cancer specimens demonstrated an increase in HDAC3 expression compared to benign tissues adjacent to the tumor (Fig. 1D-a). Survey of the benign tissue revealed mostly typical moderately differentiated adenocarcinoma (Fig. 1D-b). In diffused-type gastric cancer tissues (Fig. 1D-c), adenocarcinoma with lymph node and distant metastasis was the dominant finding (Fig. 1D-d). The percentage of positive tumor cells and staining intensity for each sample were recorded. These indicated that the level of HDAC3 expression closely correlated with increased clinical stage, as well as with lymph node and distant metastasis of the tumor-node-metastasis (TNM) classification.

\section{Pharmacological characterization of [ $\left.{ }^{3} \mathrm{H}\right]$-Honokiol binding to HDAC3.}


To investigate whether Honokiol target HDAC3, using binding affinity and DSC to verify pharmacological characterization. Binding of $\left[{ }^{3} \mathrm{H}\right]$-Honokiol to HEK-293 cell containing the recombinant human HDAC3 in the initial biological tissue linearity studies using the MES buffer is depicted in Fig. 2A. Specific ligand binding assay was closely related from total binding with this whole sample preparation, as the per cent specific binding values were $97.8 \pm 0.8 \%(n=6)$ across the throughout titration range.

Moreover, using DSC, to show shifts of protein melting temperature $(\mathrm{Tm})$ that occurs upon ligand binding to a protein gives a stabilized complex. To characterize the binding of Honokiol to HDAC3, DSC was used to unfold HDAC3 in the absence and presence Honokiol $(0.1 \mathrm{mM}$ and $0.2 \mathrm{mM})$. Data shown that Honokiol bound with the greatest affinity to the nucleotide-binding domain (NBD) as decided by the relative changes in the melting temperature $(\mathrm{Tm})$. A shift of the denaturation peak to higher temperatures in the presence of a ligand is a sign of protein-ligand binding. The changes in Tm implying that Honokiol were bound to the unfolded form of HDAC3. The purified HDAC3 proteins underwent thermal transitions, and a typical thermogram of unliganded HDAC3 is also shown in Fig. 2B. These ligand-protein binding specificity study findings proved direct and convincing evidence of efficacy that Honokiol could be directly interaction to HDAC3.

\section{Honokiol inhibited the activity and Tyr (Y298) phosphorylation of HDAC3 in gastric cancer cells}

To determine the effects of Honokiol on HDAC3 activities in vitro, AGS and SCM1 cells were treated with Honokiol $(10-60 \mu \mathrm{M})$ or with Trichostatin A (TSA; an inhibitor of HDAC) for $4 \mathrm{~h}$. Honokiol showed a significant inhibition of HDAC3 activity in a dose-dependent manner compared to vehicle control (Fig. 3A).

We nest investigated the evolutionary sequence conservation using orthologous proteins. Sequences alignment was the amino acids for residues $241-320$ of the HDAC3 proteins (Fig. 3B). Results showed that key residues within the HDAC3 catalytic site interacted with Honokiol and bound in the active site tunnel (Figs. 3C and 3D). Crystal structures of HDACs revealed that the highly conserved Tyr residue (Y298 in HDAC3) was located within the active site (Fig. 3E) in which was catalytically required in stabilizing the molecular functional activity. Changes in both conformation and dynamics might occur when Honokiol was bound to HDAC3, facilitating Honokiol targeting of the active site. This was catalytically essential in stabilizing the tetrahedral intermediate and polarizing the substrate carbonyl group (Amorati et al., 2015), resulting in blocked the HDAC3 activity (Lombardi et al., 2011; Sun et al., 2013; Watson et al., 2012).

Furthermore, specific antibody targeting Tyr (Y298) phosphorylation of HDAC3 in three gastric cancer cell lines was markedly reduced by Honokiol after $2 \mathrm{~h}$ treatment (Fig. 3F). Taken together, these results indicated the possible interaction between HDAC3 and Honokiol via structure molecular docking as well as HDAC3 kinase functional activity. Based on the above mentioned Fig. 2 and Fig. 3, evidence identify that Honokiol is a direct target of HDAC3. 
Honokiol-induced ER stress inhibited the activities of NFKB and CEBP/ $\beta$ to restrain the HDAC3 activation in gastric cancer cells(Amorati et al., 2015)

HDAC3 includes numerous transcription factors such as NF-KB and C/EBP $\beta$ binding sites (Paz-Priel et al., 2011; Somech et al., 2005). Accordingly, promoter regulation in the NF-KB and C/EBP $\beta$ promoter-flanking region (-465 - 460 and $-21 \sim-14$, individually) containing the cis-acting elements DNA binding activity in silico was also hypothesized (Fig. 4A). A previous serial study showed a Honokiol-induced ER stress cascade and activated Calpain-II activity and protein expression (Liu et al., 2012). To determine if Honokiol-induced ER stress inhibited NF-KB and C/EBP $\beta$ signals, the transcriptional activity was measured using a luciferase reporter assay (Fig. 4B). Exposure to Honokiol effectively prevented the activities of NF-KB (Fig. 4B, left panel) and C/EBPß (Fig. 4B, right panel), which could be reversed by 4phenylbutyric acid (4-PBA; a chemical chaperone) and gene silencing of Calpain-2 (shCalp-2). Results showed that both NF-KB and C/EBP $\beta$ were downstream regulated by Honokiol treatment and this was associated with ER stress induction. These results suggest that ER stress plays an important role in the regulation of transcriptional activities of transcription factor NF-KB and C/EBP $\beta$ axis in gastric cancer cells in response to Honokiol.

In addition, Honokiol treatment decreased the HDAC3 activity in AGS or SCM1 cells, which could be reversed by the over-expression of C/EBP $\beta$ (ov-C/EBP $\beta$ ) and NF-KB (ov-NF-kB) transfection compared to those in control pcDNA3 cells (Fig. 4C, left panel). Positive control by Melatonin (Wu et al., 2014; Wu et al., 2016) treatment in both ov-C/EBP $\beta$ and ov-NF-KB-transfected cells compared to control cells was also statistically confirmed (Fig. 4C, right panel). These findings indicated that Honokiol reduced NF-KB and $\mathrm{C} / \mathrm{EBP} \beta$ activation resulting in the down-regulation of HDAC3 activity. This may have potential in the development of treatment strategies for peritoneal dissemination of gastric cancer.

\section{High expression of NF-KB-p65, C/EBP $\beta$, and HDAC3 decreased overall survival probability in gastric cancer}

Using Web-based applications to conduct KM plot, univariate hazard ratio, landmark analysis, quantile survival analysis, and competing risk analysis, there was significantly positive correlations between the high expression of NF-KB-p65 (Rel A), C/EBP $\beta$, and HDAC3 in gastric cancer and overall survival and relapse free survival, as annotated in the GSE34942 for NF-KB-p65 (RELA) and GSE62254 for C/EBPB datasets by TCGA as presented in the PROGgeneV2 (Figs. 5A and 5B, respectively). Both NF-KB and $\mathrm{C} / \mathrm{EBP} \beta$ were also confirmed in patterns found in 876 patients with gastric cancer represented in the KM plot software. The public database and web application KM plotter (Suppl. Figures 1A and 1B, respectively) and the SurvExpress Web resource created the Kaplan-Meier curves based on the NF-KB-p65 (RELA), C/EBPB, and HDAC3 expression levels (Suppl. Figure 2). Taken together, the findings suggested that NF-kB-p65, C/EBP $\beta$, and HDAC3 were constitutively active in human metastatic gastric adenocarcinoma. Pharmacologic reduction of NF-KB-p65, C/EBP $\beta$, and HDAC3 expression might be useful surrogate markers for treatment activity. 
Exposure of gastric cancer cells AGS or SCM-1 to Honokiol or Tunicamycin (positive control; an ER stress inducer) led to a marked 2.3- to 5.8-fold increase of ERSE Luciferase activity compared to control cells (Fig. 6A). These cells were identified unequivocally as evidence of ER stress induction, which was further confirmed by qualitative ultrastructure analysis using transmission electron microscopy (TEM) analysis for cell organelle structure morphology. In control cells, abundant strands of rough ER with narrow cisterns were surveyed (Fig. 6B-a, arrows). However, after $12 \mathrm{~h}$ Honokiol treatment, a large part of the cisterns showed dilated organelles and were swollen into circular shapes (Fig. 6B-b, arrows). There was an unexpected finding in the HDAC3 gene silencing (shHDAC3) group (Fig. 6B-d) presenting with a similar contour of dilated organelles in ER imaging compared to control (shEGFP) group (Fig. 6B-c). These results correlated with previous findings of network regulation and control of ER stress by Honokiol exposure.

\section{Honokiol inhibited EMT markers expression and cell invasion via NF-KB-p65 and C/EBPB inhibition in gastric cancer cells}

Honokiol effectively down-regulated the protein expression of HDAC3, EMT markers (Snail, Slug, and Twist), CEBP/ $\beta$, and phosphorylated NF-KB-p65, but not phosphorylated NF-KB-p52 or p50, and markedly up-regulated ER stress-related markers (phosphorylated elf2a and GADD153) in gastric cancer cells (Fig. 7A). Knockdown HDAC3 by gene silencing could also suppress EMT markers protein expression and increase Occludin (an epithelial marker), but not E-cadherin (another epithelial marker), protein expression (Fig. 7B). Hence, we further examined whether E-cadherin distribution exchanged using immunohistochemistry analysis. Exposed to Honokiol, HDAC3 inhibitor RGFP966, and gene silencing of HDAC3 markedly increased the expression of phosphorylated E-cadherin, but decreased the expression of $\beta$ catenin in SCM1 cells (Fig. 7C). We next examined whether knockdown of NF-KB-p65 and C/EBP $\beta$ participated in the EMT process in gastric cancer cells. Results revealed that TGF $\beta$ induced the changes of morphologic characteristics of epithelial cells, from the typical cobblestone type to the slender fibroblast-like, or exhibited a single spindle-shaped cell phenotype, which could be effectively reversed by the treatments with Honokiol, melatonin, and gene silencing of NF-kB-p65 or C/EBPB (Fig. 7D). We further verified the cell invasion effect by Honokiol. Results showed that Honokiol, melatonin, and gene silencing of NF-kB-p65 or C/EBP $\beta$ markedly suppressed TGF $\beta$-induced cell invasion (Fig. 7E). These results suggest that Honokiol is capable of inhibiting cell invasion via inhibition of NF-KB-p65 and C/EBP $\beta$ signals.

\section{Honokiol impeded cell migration and invasion via HDAC3 suppression in gastric cancer cells}

To investigate if HDAC3 could confer enhanced migratory and invasive capacities to gastric cancer cells, the migration and invasion was assessed using two distinct shRNAs targeting HDAC3 (shHDAC3 \#267; \#828) and the pharmacologic HDAC3 inhibitor RGFP966. As controls, cells were transduced encoding a non-targeting shRNA (shEGFP). The efficiency of the shHDAC3-mediated silencing was confirmed by RTqPCR, immuno-blotting, and HDAC3 activity (Supplementary Fig. 3). Honokiol, melatonin, RGF966, and shRNAs targeting HDAC3 significantly impaired cell migration (Figs. 8A-8B) and invasion (Figs. 8C-8D). 
Efficacy was confirmed by monitoring the number of viable cells. In both assays, HDAC3 silencing reduced the capacity of gastric cancer cells to migrate by more than $60 \%$. Transwell invasion assays further revealed significant impairment of gastric cancer cells to invade through the matrigel-coated filters when HDAC3 was silenced.

To explore the involvement of Wnt/ $\beta$-catenin in ER stress-reduced peritoneal cavity, Wnt/ $\beta$-catenin signaling was examined in gastric cancer cells. Results revealed that Honokiol significantly decreased the nuclear localization of $\beta$-catenin (data not shown). Honokiol also decreased the TopFlash activity for Wnt/ $\beta$-catenin signaling (Fig. 8E). The gene knockdown of NFKB-p65, C/EBP $\beta$, or HDAC3 combined with/without Honokiol treatment could also significantly decrease the TopFlash activity (Fig. 8E). Interestingly, shNFkB-p65, shC/EBP $\beta$, or shHDAC3 prevented the down-regulation of TopFlash activity induced by the knockdown per se, suggesting that Wnt/ $\beta$-catenin may be a target of NFKB-p65, C/EBP 3 , or HDAC3 in vitro. These results also indicated that Honokiol significantly suppressed mesenchymal characteristics, increased the expression of epithelial signature markers, and suppressed Wnt expression through NFKB-p65, C/EBPß, or HDAC3-dependent regulation, which affected cancer cell migration and invasion in vitro.

\section{Honokiol and gene silencing HDAC3 restrained gastric tumor growth and peritoneal dissemination in a mouse model}

Honokiol administration ( $5 \mathrm{mg} / \mathrm{kg} /$ twice/weekly) inhibited tumor growth, metastatic peritoneal extension, and macro-metastasis by $80-85 \%$ compared to control group detected by PET/CT imaging and quantification (Figs. 9A and 9B) and macroscopic examination of the tumor burden (Fig. 9D) along with major organ metastasis (Supplementary Fig. 4). Quantifications of estimated radioactivity $(\mathrm{Bq} / \mathrm{ml}$ ) and specific uptake values (SUV), which were as indices to determine if a hotspot was significant, were calculated. Gene silencing HDAC3 treatment repressed intestinal mesenteric nodules and had diverse organs effects similar to those of the pharmacologic inhibitor RGFP966 (Supplementary Fig. 4). Quantification of parietal peritoneum metastasis of gastric tumor cells by Honokiol and gene silencing HDAC3 revealed that Honokiol had no toxic effects and had modest gains in body weight. The quantification of body weight, tumor weight, and number of nodules were shown in Figs. 9C-9F, respectively. Histological examination for animal tumor tissues revealed high expressions of E-cadherin phosphorylation in the groups of Honokiol and gene silencing HDAC3 (Fig. 9G). These results indicated that Honokiol therapy led to a substantial diminution in gastric tumor growth and metastatic dissemination in an experimental mouse model.

\section{Discussion}

The results here support the hypothesis that HDAC3 plays a crucial role in suppressing gastric cancer cell metastatic dissemination. These processes, in turn, can be modulated by Honokiol-induced ER stress. Honokiol also promotes calpain activation by targeting NF-KB-p65 and C/EBP $\beta$, thereby inhibiting the effects of HDAC3. Moreover, HDAC3 is significantly over-expressed in both gastric tissues and gastric 
cancer cell lines. Histopathologic investigation of human gastric cancer tissues reveals a significant correlation between HDAC3 expression and clinical staging, expressed in the TNM (Tumor-NodeMetastasis) classification. In the analysis of verified clinical samples of the omentum, distal, and lymph node metastases, high HDAC3 expression with markedly dense staining is a poor prognostic indicator in patients with gastric cancer. In animal experiments, HDAC3 over-expression in tumor mass can be significantly inhibited by Honokiol treatment. Further, the down-regulation of HDAC3 through gene silencing markedly blocks the metastatic capability of gastric cancer cells in nude mice, especially towards the abdominal cavity, liver, and lungs. These findings demonstrate HDAC3 detection may provide clear and realistic explanations regarding useful information, while histopathologic studies may provide prognostic stratification and help in choosing appropriate treatment strategies. (Fig. 10)

Class I histone deacetylase HDAC3 is involved in "epigenetic" gene regulation through the control of the acetylation state of lysine side chains in histone tails. HDAC3 is also a component of the NCoR-SMRT corepressor complex, which is different from co-repressor complexes that typically contain HDAC1 and 2 (Watson et al., 2016). A previous report shows that the HDAC3 protein has common features with HDAC1 and HDAC2, namely deacetylation of histone substrates and transcriptional repression in early functional analysis (Karagianni and Wong, 2007; Yang et al., 1997). In contrast, HDAC3 includes an intriguingly variable $\mathrm{C}$ terminus, with no apparent similarity to other HDACs, indicating that HDAC3 may have some unique properties and may not be completely redundant with other HDACs. Moreover, advanced functional domain analysis reveals that HDAC3 contains both nuclear export and import signals, which account for its distinct localization pattern (Yang et al., 2002). The subcellular distribution of HDAC3 supports its nuclear function and uncovers a whole new world of potential cytoplasmic substrates and regulators. Initial implications of HDAC3 in tumorigenesis and carcinogenesis have been based on correlative studies reporting aberrant expression and/or localization of HDAC3 in various tumors (Bartling et al., 2005). Elevated and deregulated HDAC3 expression is also found in human astrocytic glial tumors (Liby et al., 2006). Gene silencing of HDAC3 in human colon cancer results in cell growth inhibition, differentiation, and increased apoptosis (Wilson et al., 2006). From a mechanistic perspective, both epithelial and mesenchymal cells respond to hypoxia with an HIF-dependent induction of HDAC3. In the epithelia, increased HDAC3 deacetylates H3K4ac to restrain the chromatin structure and expression of epithelial genes. At the same time, WDR5 and HDAC3 levels are increase in mesenchymal cells. A previous study has shown that an interplay between HDAC3 and WDR5 collectively regulates low levels of oxygen-induced EMT (Mani and Barton, 2011). Furthermore, the tumor antigen MAGE-A can recruit HDAC3 to impair the trans-activation function of the tumor suppressor p53. This confers resistance to chemotherapeutic agents (Monte et al., 2006). Based on study with human maxillary carcinoma cells under acidic conditions, HDAC3 inhibition combined with hyperthermia may provide an efficient therapeutic approach for cancer (Narita et al., 2005).

Previous studies have shown a contributing role of HDAC3 in a compendium of clinical cancers. However, the role of HDAC3 in cancers is controversial. In a study, HDAC3 depletion substantially increased the migration of MDA-MB-231 metastatic breast cancer cells (Kim et al., 2010). Loss of HDAC3 could lead to hepatocellular carcinoma (Bhaskara et al., 2010), while another study found that the increased HDAC3 
levels are associated with the improved patient survival (Junqueira-Neto et al., 2015). In line with results reported by Wu et al. (2011), knockdown of endogenous HDAC3 caused a complete and significant loss of EMT and inhibits the migration and invasion activity in FADU or MCF-7 clones. A critical role of HDAC3 in facilitating the control of EMT by different EMT regulators has been suggested (Mani and Barton, 2011; Wu et al., 2011). Similarly, the current study also revealed that HDAC3 could interact with different EMT regulators to mediate E-cadherin repression in gastric cancer cells. Indeed, the immunohistochemical evidence is consistent with the aforementioned findings and demonstrates that the increased HDAC3 protein levels lead to worse clinical outcomes in patients with gastric cancer.

The EMT contributes to early metastasis, tumor progression, and resistance to treatments, indicating the development of cancer aggressiveness, growth, and spread in the early stages of the disease, although the role of EMT in metastasis is a longstanding source of debate (Cheung et al., 2013; Fischer et al., 2015; Zheng et al., 2015). Understanding the regulatory factors for EMT may help in the development of antimetastasis therapeutics, anti-chemoresistance, as well as diagnostic or prognostic markers for various tumors. However, the effects of HDACs on EMT are also controversial. Numerous studies have demonstrated that HDACs inhibit EMT but this is not always the case. It is noteworthy that HDAC inhibitors might reinforce EMT progression (Debeb et al., 2012; Ji et al., 2015; Kong et al., 2012; Sakamoto et al., 2016; Wawruszak et al., 2019a; Wawruszak et al., 2019b; Wu et al., 2016a). Although the role of different HDACs in mediating EMT are well documented, whether or not a transcription-regulated HDAC3 is required to coordinate different EMT regulators remains unknown. As previously reported by Qi et al. (2008), HDAC3 is related to Snail signaling and HDAC inhibitors impair Snail-mediated transcriptional repression in cells during early Drosophila development (Qi et al., 2008). In addition, Zeng and colleagues (2013) have demonstrated that HDAC3 mRNA can undergo unconventional splicing to modulate HDAC3 and induce EMT via transforming growth factor $\beta 2$ (TGF $\beta 2$ ) (Zeng et al., 2013). Zhang L et al. (2019) have also reported that HDAC3 down-regulation by ginsenoside Rg3 inhibits the EMT of cutaneous squamous cell carcinoma via c-Jun acetylation (Zhang et al., 2019). Recently, I-7ab has been found to inhibit the growth of triple negative breast cancer (TNBC) cells via targeting HDAC3 and promoting p53 acetylation (Yang et al., 2018). The miRNA regulates hypoxia-induced EMT and metastasis by repressing HDAC3 and SENP1 expression and by presenting a regulatory network that involves many key players in hypoxia-induced EMT (Chen et al., 2016). The RORa/HDAC3-mediated attenuation of NF-kB signaling controls the balance of inflammatory responses, suggesting that therapeutic strategies targeting this epigenetic regulation may be beneficial in the treatment of chronic inflammatory diseases, including inflammatory bowel disease (IBD) (Oh et al., 2019). Wang et al. (2016) showed that disrupting the CBX4HDAC3 interaction abolished the Runx2 inhibition, as well as the inhibition of cell migration and invasion (Wang et al., 2016). Clinically, compared to normal adjacent tissues, esophageal tumor samples show an up-regulation of SOX4, EZH2, and HDAC3; the EZH2 expression is significantly increased in metastatic ESCC tissues (Koumangoye et al., 2015). SOX4 promotes esophageal tumor cell proliferation and invasion by silencing miR-31 via the activation and stabilization of a co-repressor complex with EZH2 and HDAC3 (Koumangoye et al., 2015). In the present study, the findings suggest a crucial role for HDAC3 
in suppressing E-cadherin transcription and in up-regulating mesenchymal markers during EMT in gastric cancer.

\section{Summary And Conclusion}

The present study demonstrates that HDAC3 can regulate E-cadherin expression and EMT through the transcription factors $C / E B P \beta$ and NFKB. Our data also reveals that HDAC3 knockdown down-regulates the expression of $\mathrm{Wnt} / \beta$-catenin signaling. To determine if transcription factors $\mathrm{C} / \mathrm{EBP} \beta$ and NFKB have any causal role in Wnt/ $\beta$-catenin signaling, silencing of $C / E B P \beta$ and NFKB individually was done. The results showed that the $\mathrm{Wnt} / \beta$-catenin activity by TopFlash luciferase assay was decreased in gastric cancer cells with gene silencing of C/EBP $\beta$ and NFKB-p65, indicating that Wnt/ $\beta$-catenin may be a target for NFKB-p65 or C/EBP $\beta$. The pharmacologic inhibition or gene silencing of HDAC3 could also suppress the migration and metastasis in gastric cancer cells in vitro and in vivo. The experimental animal model also proves a HDAC3 knockdown-mediated epithelial morphology accompanied with increased Ecadherin, suggesting a pathologic function of HDAC3 regulation in EMT expression. Nevertheless, how HDAC3 can regulate the expression of these EMT-inducing transcription factors and Wnt/ $\beta$-catenin signaling in gastric cancer cells warrants further investigation. Overall, this study demonstrates that HDAC3 may be a positive regulator of EMT and tumor metastasis in gastric cancer through transcriptional repression of E-cadherin via C/EBP $\beta$ and NFKB-p65 gene regulation, which can be significantly inhibited by treatment with Honokiol or HDAC3 inhibitors. Thus, HDAC3 may be a potential therapeutic target for reversing EMT and tumor metastasis in gastric cancer progression.

\section{Data Availability Statement}

Data available on request from the authors. The data that support the findings of this study are available from the corresponding author upon reasonable request. Some data may not be made available because of privacy or ethical restrictions.

\section{Abbreviations}

GC Gastric cancer

HDIs Histone deacetylase inhibitors

HDAC3 Histone deacetylase 3

EMT Epithelial-mesenchymal transition

ER Endoplasmic reticulum

C/EBP $\beta$ CCAAT/enhancer-binding protein B

NF-KB Nuclear factor kappa-light-chain-enhancer of activated B cells 
ERSE ER stress response element

TEM Transmission electron microscope

PET/CT Positron emission tomography-computed tomography

shRNA Short hairpin RNA

DSC Differential Scanning Calorimetry

NBD Nucleotide-binding domain

Tm Melting temperature

\section{Declarations}

\section{Acknowledgements}

The authors wish to thank Taichung Veterans General Hospital (TCVGH), particularly its Tissue Bank, for the human gastric cancer specimens, for the HDAC3 staining, and for its Animal Center Care. The authors also thank the National RNAi Core Facility Platform, Taipei, Taiwan for the shRNA and the Genome Research Center, National Yang-Ming University for the pcDNA.

\section{Funding statement}

This work was supported by grants from the Ministry of Science and Technology, the National Science Council, Taiwan (MOST-106-2320-B-005-001-MY3, MOST-104-2320-B-005-002-MY3), National Chung Hsing University, Taiwan (NCHU108ST001F), and Taichung Veterans General Hospital in Taiwan (TCVGH1087327D, TCVGH-1087308C, TCVGH-1077311C, TCVGH-1077329D).

\section{Author Contributions}

S.-M Wu and C.-N Yang performed all of the mouse and cellular experiments, and generated and analyzed data; Y.-J Jan performed the pathological diagnosis; C.-S Chiu and Meng M performed binding assay and DSC. S.-C Tsai performed the PET/CT study; J.-L. Arbiser conceived the study; C.-C Shen carried out the molecular docking; S.-H Liu and H.-C Pan conceived the study, interpretation of data, and drafting and critical revision of the manuscript; M.-L. Sheu designed the experiments, analyzed the data, and wrote the manuscript. All authors had access to the study data and reviewed and approved the final manuscript.

\section{Ethics approval}


The study was approved by the Institutional Review Board of the Taichung Veterans General Hospital Taiwan and the study conformed to the principles outlined in the Declaration of Helsinki (Approval No. 17096B). All experimental procedures and protocols involved in the present study were approved by the appropriate Institutional Animal Care and Use Committee (IACUC) (Approval No La-1061488) by Taichung Veterans General Hospital Taiwan.

Consent to participate No application.

Consent for publication All authors have agreed to publish this manuscript.

Code availability No application.

Conflict of interest The authors declare no competing interests.

\section{References}

1. Amorati R, Zotova J, Baschieri A, Valgimigli L. Antioxidant Activity of Magnolol and Honokiol: Kinetic and Mechanistic Investigations of Their Reaction with Peroxyl Radicals. J Org Chem. 2015;80:10651-59.

2. Bartling B, Hofmann HS, Boettger T, Hansen G, Burdach S, Silber RE, et al. Comparative application of antibody and gene array for expression profiling in human squamous cell lung carcinoma. Lung Cancer (Amsterdam Netherlands. 2005;49:145-54.

3. Bhaskara S, Knutson SK, Jiang G, Chandrasekharan MB, Wilson AJ, Zheng S, et al. Hdac3 is essential for the maintenance of chromatin structure and genome stability. Cancer Cell. 2010;18:436-47.

4. Brabletz T. To differentiate or not-routes towards metastasis. Nat Rev Cancer. 2012;12:425-36.

5. Brabletz T, Kalluri R, Nieto MA, Weinberg RA. EMT in cancer. Nat Rev Cancer. 2018;18:128-34.

6. Chen SY, Teng SC, Cheng TH, Wu KJ. miR-1236 regulates hypoxia-induced epithelial-mesenchymal transition and cell migration/invasion through repressing SENP1 and HDAC3. Cancer Lett. 2016;378:59-67.

7. Cheung KJ, Gabrielson E, Werb Z, Ewald AJ. Collective invasion in breast cancer requires a conserved basal epithelial program. Cell. 2013;155:1639-51.

8. Chiu CS, Tsai CH, Hsieh MS, Tsai SC, Jan YJ, Lin WY, Lai DW, Wu SM, Hsing HY, Arbiser JL, Sheu ML. Exploiting Honokiol-induced ER stress CHOP activation inhibits the growth and metastasis of melanoma by suppressing the MITF and beta-catenin pathways. Cancer Lett. 2019;442:113-25.

9. Davis TL, Sharif NA. Pharmacological characterization of $[(3) \mathrm{H}]$-prostaglandin $\mathrm{E}(2)$ binding to the cloned human EP(4) prostanoid receptor. Br J Pharmacol. 2000;130:1919-26.

10. Debeb BG, Lacerda L, Xu W, Larson R, Solley T, Atkinson R, et al. Histone deacetylase inhibitors stimulate dedifferentiation of human breast cancer cells through WNT/ $\beta$-catenin signaling. Stem Cells (Dayton Ohio). 2012;30:2366-77. 
11. Emmett MJ, Lazar MA. Integrative regulation of physiology by histone deacetylase 3. Nat Rev Mol Cell Biol. 2019;20:102-15.

12. Fischer KR, Durrans A, Lee S, Sheng J, Li F, Wong ST, et al. Epithelial-to-mesenchymal transition is not required for lung metastasis but contributes to chemoresistance. Nature. 2015;527:472-76.

13. Fried LE, Arbiser JL. Honokiol, a multifunctional antiangiogenic and antitumor agent. Antioxid Redox Signal. 2009;11:1139-48.

14. Gherardi E, Birchmeier W, Birchmeier C, Vande Woude G. Targeting MET in cancer: rationale and progress. Nat Rev Cancer. 2012;12:89-103.

15. Ji M, Lee EJ, Kim KB, Kim Y, Sung R, Lee SJ, et al. HDAC inhibitors induce epithelial-mesenchymal transition in colon carcinoma cells. Oncol Rep. 2015;33:2299-308.

16. Jung HY, Fattet $L$, Yang J. Molecular pathways: linking tumor microenvironment to epithelialmesenchymal transition in metastasis. Clin Cancer Res. 2015;21:962-68.

17. Junqueira-Neto S, Vieira FQ, Montezuma D, Costa NR, Antunes L, Baptista T, et al. Phenotypic impact of deregulated expression of class I histone deacetylases in urothelial cell carcinoma of the bladder. Mol Carcinog. 2015;54:523-31.

18. Juttner S, Wissmann C, Jons T, Vieth M, Hertel J, Gretschel S, et al. Vascular endothelial growth factor-D and its receptor VEGFR-3: two novel independent prognostic markers in gastric adenocarcinoma. J Clin Oncol. 2006;24:228-40.

19. Karagianni P, Wong J. HDAC3. taking the SMRT-N-CoRrect road to repression. Oncogene. 2007;26:5439-449.

20. Kim HC, Choi KC, Choi HK, Kang HB, Kim MJ, Lee YH, et al. HDAC3 selectively represses CREB3mediated transcription and migration of metastatic breast cancer cells. Cellular molecular life sciences: Cell Mol Life Sci. 2010;67:3499-510.

21. Kong D, Ahmad A, Bao B, Li Y, Banerjee S, Sarkar FH. Histone deacetylase inhibitors induce epithelialto-mesenchymal transition in prostate cancer cells. PLoS One. 2012;7:e45045.

22. Koumangoye RB, Andl T, Taubenslag KJ, Zilberman ST, Taylor CJ, Loomans HA, et al. SOX4 interacts with EZH2 and HDAC3 to suppress microRNA-31 in invasive esophageal cancer cells. Mol Cancer. $2015 ; 14: 24$.

23. Lai DW, Liu SH, Karlsson Al, Lee WJ, Wang KB, Chen YC, et al. The novel Aryl hydrocarbon receptor inhibitor biseugenol inhibits gastric tumor growth and peritoneal dissemination. Oncotarget. 2014;5:7788-804.

24. Lee WJ, Lan KH, Chou CT, Yi YC, Chen WC, Pan HC, et al. Tpl2 inhibitors thwart endothelial cell function in angiogenesis and peritoneal dissemination. Neoplasia (New York NY). 2013;15:1036-48.

25. Liby P, Kostrouchova M, Pohludka M, Yilma P, Hrabal P, Sikora J, et al. Elevated and deregulated expression of HDAC3 in human astrocytic glial tumours. Folia Biol (Praha). 2006;52:21-33.

26. Liu SH, Lee WJ, Lai DW, Wu SM, Liu CY, Tien HR, et al. Honokiol confers immunogenicity by dictating calreticulin exposure, activating ER stress and inhibiting epithelial-to-mesenchymal transition. Mol 
Oncol. 2015;9:834-49.

27. Liu SH, Shen CC, Yi YC, Tsai JJ, Wang CC, Chueh JT, et al. Honokiol inhibits gastric tumourigenesis by activation of 15-lipoxygenase-1 and consequent inhibition of peroxisome proliferator-activated receptor-gamma and COX-2-dependent signals. Br J Pharmacol. 2010;160:1963-72.

28. Liu SH, Wang KB, Lan KH, Lee WJ, Pan HC, Wu SM, et al. Calpain/SHP-1 interaction by honokiol dampening peritoneal dissemination of gastric cancer in nu/nu mice. PLoS One. 2012;7:e43711.

29. Lombardi PM, Cole KE, Dowling DP, Christianson DW. Structure, mechanism, and inhibition of histone deacetylases and related metalloenzymes. Curr Opin Struct Biol. 2011;21:735-43.

30. Ma Y, Duan J, Hao X. Down-regulated HDAC3 elevates microRNA-495-3p to restrain epithelialmesenchymal transition and oncogenicity of melanoma cells via reducing TRAF5. J Cell Mol Med. 2020;24:12933-44.

31. Maehara Y, Kakeji Y, Kabashima A, Emi Y, Watanabe A, Akazawa K, et al. Role of transforming growth factor-beta 1 in invasion and metastasis in gastric carcinoma. J Clin Oncol. 1999;17:607-14.

32. Mani SA, Barton MC. HDAC3 at the fulcrum of an epithelial-mesenchymal balance. Mol Cell. 2011;43:697-98.

33. Martin S, Lamb HK, Brady C, Lefkove B, Bonner MY, Thompson P, et al. Inducing apoptosis of cancer cells using small-molecule plant compounds that bind to GRP78. Br J Cancer. 2013;109:433-43.

34. Monte M, Simonatto M, Peche LY, Bublik DR, Gobessi S, Pierotti MA, et al. MAGE-A tumor antigens target p53 transactivation function through histone deacetylase recruitment and confer resistance to chemotherapeutic agents. Proc Natl Acad Sci U S A. 2006;103:11160-65.

35. Narita N, Fujieda S, Tokuriki M, Takahashi N, Tsuzuki H, Ohtsubo T, et al. Inhibition of histone deacetylase 3 stimulates apoptosis induced by heat shock under acidic conditions in human maxillary cancer. Oncogene. 2005;24:7346-54.

36. Oh SK, Kim D, Kim K, Boo K, Yu YS, Kim IS, et al. RORalpha is crucial for attenuated inflammatory response to maintain intestinal homeostasis. Proc Natl Acad Sci U S A. 2019;116:21140-49.

37. Pan HC, Lai DW, Lan KH, Shen CC, Wu SM, Chiu CS, et al. Honokiol thwarts gastric tumor growth and peritoneal dissemination by inhibiting Tpl2 in an orthotopic model. Carcinogenesis. 2013a;34:256879.

38. Pan HC, Yang CN, Hung YW, Lee WJ, Tien HR, Shen CC, et al. Reciprocal modulation of C/EBP-alpha and C/EBP-beta by IL-13 in activated microglia prevents neuronal death. Eur J Immunol. 2013b;43:2854-65.

39. Paz-Priel I, Houng S, Dooher J, Friedman AD. C/EBPa and C/EBPa oncoproteins regulate nfkb1 and displace histone deacetylases from NF-кB p50 homodimers to induce NF-кB target genes. Blood. 2011;117:4085-94.

40. Qi D, Bergman M, Aihara H, Nibu Y, Mannervik M. Drosophila Ebi mediates Snail-dependent transcriptional repression through HDAC3-induced histone deacetylation. Embo j. 2008;27:898-909. 
41. Sakamoto T, Kobayashi S, Yamada D, Nagano H, Tomokuni A, Tomimaru Y, et al. A Histone Deacetylase Inhibitor Suppresses Epithelial-Mesenchymal Transition and Attenuates Chemoresistance in Biliary Tract Cancer. PLoS One. 2016;11:e0145985.

42. Somech R, Shaklai S, Geller O, Amariglio N, Simon AJ, Rechavi G, et al. The nuclear-envelope protein and transcriptional repressor LAP2beta interacts with HDAC3 at the nuclear periphery, and induces histone H4 deacetylation. J Cell Sci. 2005;118:4017-25.

43. Sun Z, Feng D, Fang B, Mullican SE, You SH, Lim HW, et al. Deacetylase-independent function of HDAC3 in transcription and metabolism requires nuclear receptor corepressor. Mol Cell. 2013;52:769-82.

44. Tsai JH, Yang J. Epithelial-mesenchymal plasticity in carcinoma metastasis. Genes Dev. 2013;27:2192-206.

45. Wang X, Li L, Wu Y, Zhang R, Zhang M, Liao D, et al. CBX4 Suppresses Metastasis via Recruitment of HDAC3 to the Runx2 Promoter in Colorectal Carcinoma. Cancer Res. 2016;76:7277-89.

46. Watson PJ, Fairall L, Santos GM, Schwabe JW. Structure of HDAC3 bound to co-repressor and inositol tetraphosphate. Nature. 2012;481:335-40.

47. Watson PJ, Millard CJ, Riley AM, Robertson NS, Wright LC, Godage HY, et al. Insights into the activation mechanism of class I HDAC complexes by inositol phosphates. Nat Commun. 2016;7:11262.

48. Wawruszak A, Gumbarewicz E, Okon E, Jeleniewicz W, Czapinski J, Halasa M, et al. Histone deacetylase inhibitors reinforce the phenotypical markers of breast epithelial or mesenchymal cancer cells but inhibit their migratory properties. Cancer Manag Res. 2019a;11:8345-58.

49. Wawruszak A, Kalafut J, Okon E, Czapinski J, Halasa M, Przybyszewska A, et al. Histone Deacetylase Inhibitors and Phenotypical Transformation of Cancer Cells. Cancers. 2019b;11.

50. Williams ED, Gao D, Redfern A, Thompson EW. Controversies around epithelial-mesenchymal plasticity in cancer metastasis. Nat Rev Cancer. 2019;19:716-32.

51. Wilson AJ, Byun DS, Popova N, Murray LB, L'Italien K, Sowa Y, et al. Histone deacetylase 3 (HDAC3) and other class I HDACs regulate colon cell maturation and p21 expression and are deregulated in human colon cancer. J Biol Chem. 2006;281:13548-58.

52. Wu CY, Tsai YP, Wu MZ, Teng SC, Wu KJ. Epigenetic reprogramming and post-transcriptional regulation during the epithelial-mesenchymal transition. Trends Genet. 2012;28:454-63.

53. Wu MZ, Tsai YP, Yang MH, Huang CH, Chang SY, Chang CC, et al. Interplay between HDAC3 and WDR5 is essential for hypoxia-induced epithelial-mesenchymal transition. Mol Cell. 2011;43:811-22.

54. Wu S, Luo Z, Yu PJ, Xie H, He YW. Suberoylanilide hydroxamic acid (SAHA) promotes the epithelial mesenchymal transition of triple negative breast cancer cells via HDAC8/FOXA1 signals. Biol Chem. 2016a;397:75-83.

55. Wu SM, Lin WY, Shen CC, Pan HC, Keh-Bin W, Chen YC, et al. Melatonin set out to ER stress signaling thwarts epithelial mesenchymal transition and peritoneal dissemination via calpain-mediated C/EBPbeta and NFkappaB cleavage. J Pineal Res. 2016b;60:142-54. 
56. Yang M, Dang X, Tan Y, Wang M, Li X, Li G. I-7ab inhibited the growth of TNBC cells via targeting HDAC3 and promoting the acetylation of p53. Biomed Pharmacother. 2018;99:220-26.

57. Yang WM, Tsai SC, Wen YD, Fejer G, Seto E. Functional domains of histone deacetylase-3. J Biol Chem. 2002;277:9447-54.

58. Yang WM, Yao YL, Sun JM, Davie JR, Seto E. Isolation and characterization of cDNAs corresponding to an additional member of the human histone deacetylase gene family. J Biol Chem.

1997;272:28001-007.

59. Zeng L, Wang G, Ummarino D, Margariti A, Xu Q, Xiao Q, et al. Histone deacetylase 3 unconventional splicing mediates endothelial-to-mesenchymal transition through transforming growth factor beta2. J Biol Chem. 2013;288:31853-66.

60. Zhang L, Shan X, Chen Q, Xu D, Fan X, Yu M, et al. Downregulation of HDAC3 by ginsenoside Rg3 inhibits epithelial-mesenchymal transition of cutaneous squamous cell carcinoma through c-Jun acetylation. J Cell Physiol. 2019;234:22207-19.

61. Zheng X, Carstens JL, Kim J, Scheible M, Kaye J, Sugimoto H, et al. Epithelial-to-mesenchymal transition is dispensable for metastasis but induces chemoresistance in pancreatic cancer. Nature. 2015;527:525-30.

\section{Figures}
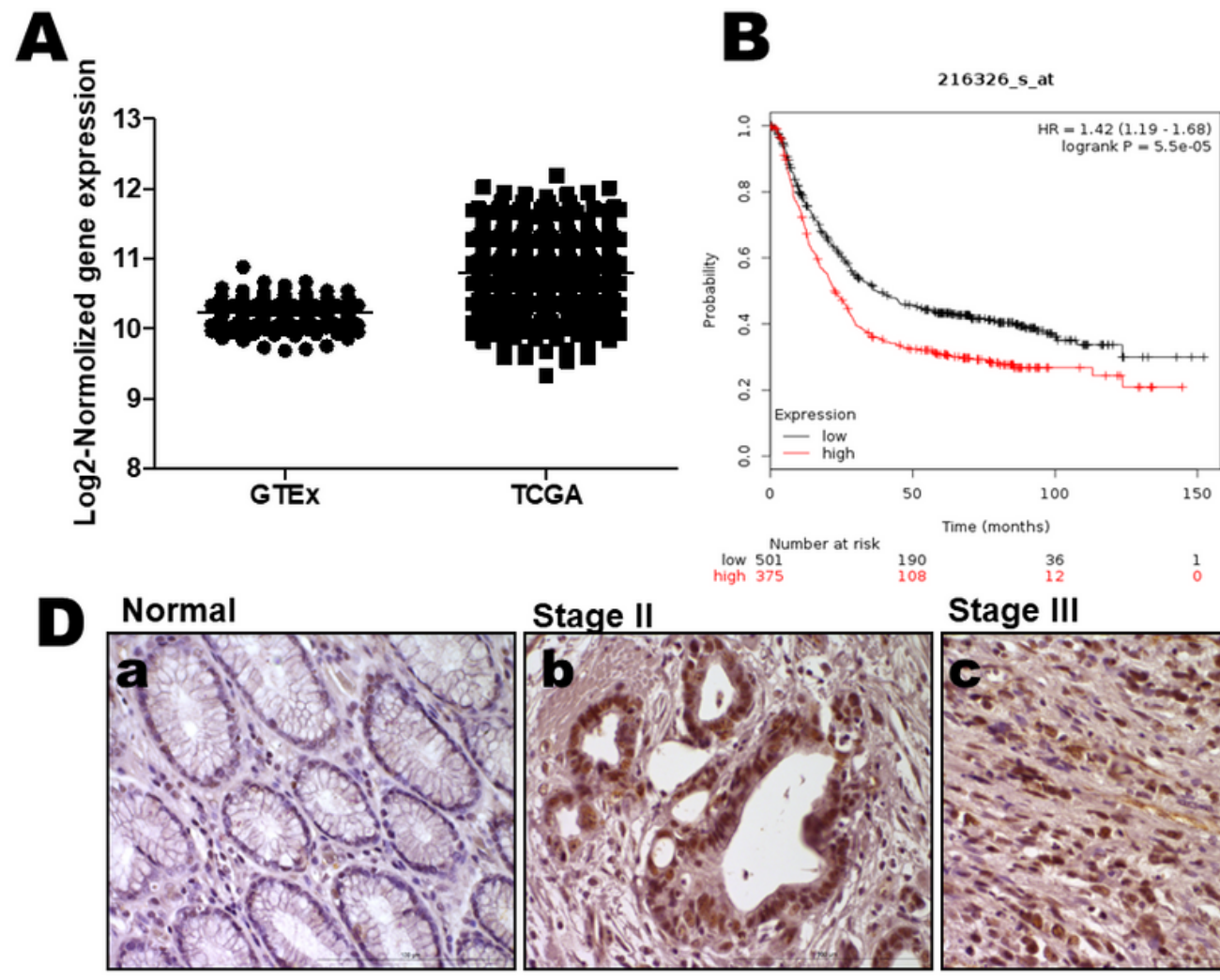

Stage III

Stage IV
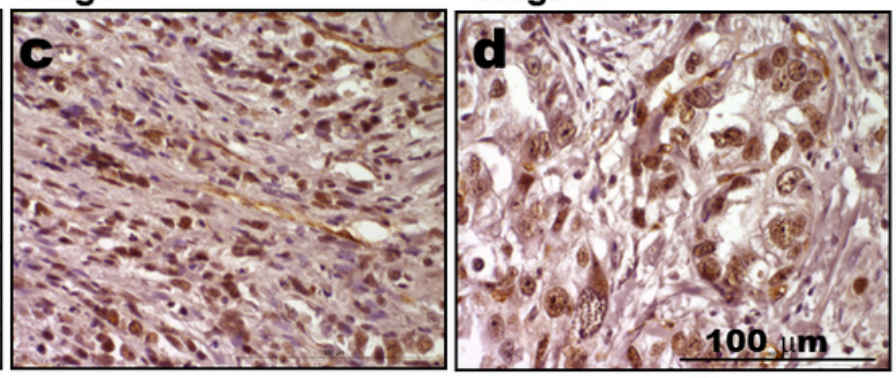

Figure. 1

Figure 1 
High HDAC3 expression in patients with gastric cancer correlated with survival rate. (A) HDAC3 expression levels in normal tissues and tumor samples derived from publicly available Genotype-Tissue Expression (GTEx, n=172) and The Cancer Genome Atlas (TCGA, $n=414)$ gene expression data plotted as box and whisker plots. (B) High HDAC3 expressions were associated with decreased overall survival probability in gastric cancer. Data obtained were from the dataset (216326_s_at, n=876) through a comprehensive search using Kaplan-Meier plotter.com for HDAC3 evaluation. (C) Enhancements of the existing database SurvExpress for HDAC3 $(n=57)$. (D) HDAC3 is up-regulated in gastric cancer tissues from the Taichung Veterans General Hospital (TCVGH) Tissues Bank. (a) Representative immunostaining of HDAC3 expressions in human normal gastric mucosa. (b) Poorly differentiated intestinal adenocarcinoma, (c) diffused adenocarcinoma in mucosa, and (d) diffuse adenocarcinoma in serosa are shown. Scale bar $=100 \mu \mathrm{m}$

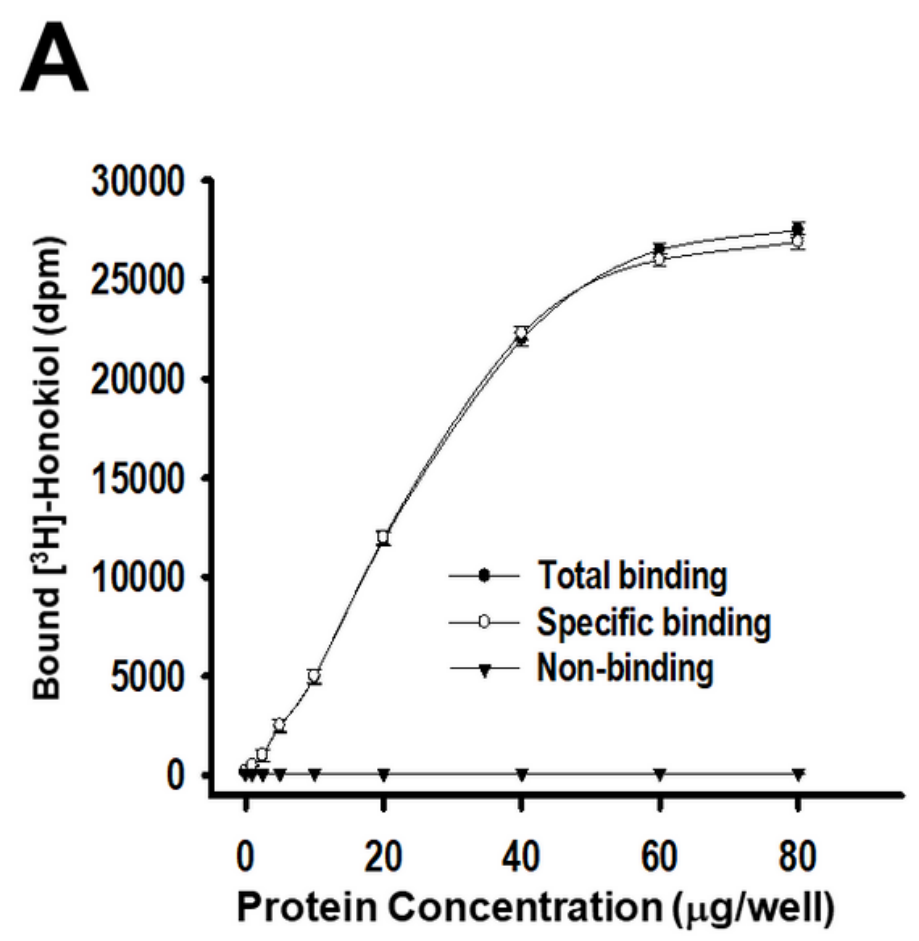

\section{B}

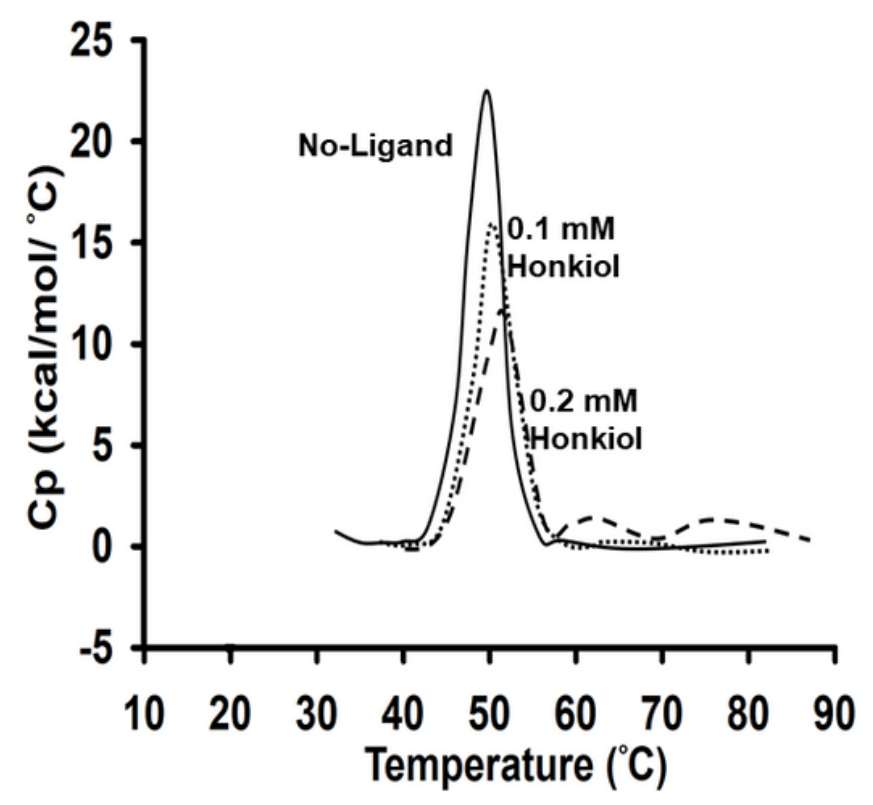

Figure. 2

\section{Figure 2}

Pharmacological characterization of [3H]-Honokiol binding to HDAC3. (A) Biological tissue linearity of [3H]-Honokiol binding to HEK-293 cell membranes expressing the recombinant human HDAC3. Sample were added in varying amounts to $0.2 \mathrm{nM}[3 \mathrm{H}]$-Honokiol in a final volume of $500 \mu$ l and the binding assay performed as described in the Methods. Data from a representative experiment is shown. Note the very high level of specific binding found in this system. (B) The purpose of this protocol is to prepare HDAC3 $(10 \mu \mathrm{M})$ in $50 \mathrm{mM}$ HEPES, $0.1 \mathrm{M} \mathrm{KCl}$ and $10 \mathrm{mM}$ beta-mercaptoethanol $\mathrm{pH} 7.2$ was contingent thermal denaturation in the presence or absence of Honokiol for DSC. Subsequently, samples were heated from $25-80^{\circ} \mathrm{C}$ at a rate of temperature $90^{\circ} \mathrm{C}$ per hour. Solid lines, no ligand; dashed lines, $0.1 \mathrm{mM}$ Honokiol; dotted lines and dashed $0.2 \mathrm{mM}$ Honokiol. The contours were essentially the same when determined in a 


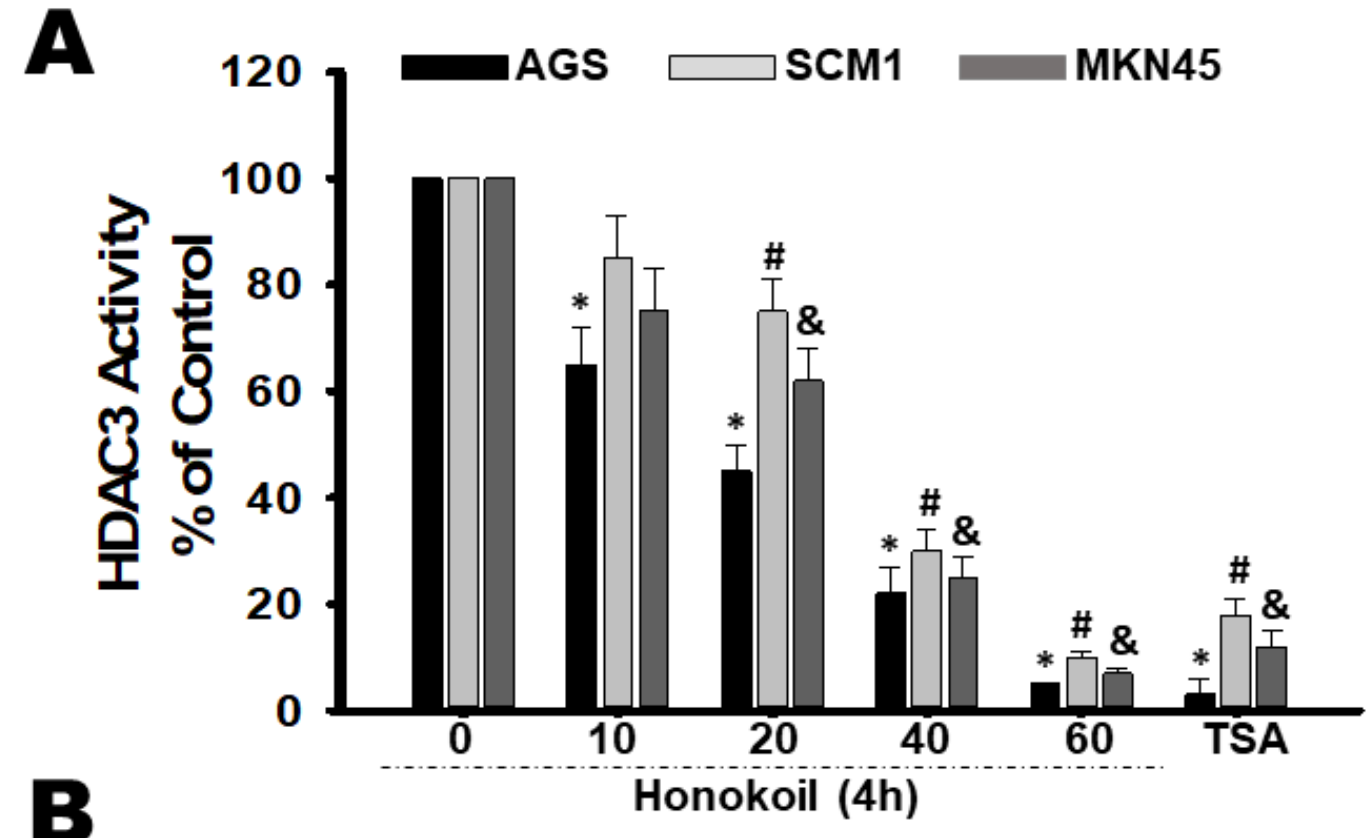

Homo sapiens 241 NQVVDFYQPTCIVLQCGADSLGCDRLGCFNLSIRGHGECVEYVKSFNIPLLVLGGGGYTVRNVARCWTYETSLLVEEAIS 320 Mus musculus 241 SQVVDFYQPTCIVLQCGAD SLGCDRLGCFNLSIRGHGECVEYVKSFNIPLLVLGGGGYTVRNVARCWTYETSLLVEEAIS 320 Rattus norvegicus 241 SQVVDFYQPXCIVLQCGAD SLGCDRLGCFNLSIRGHGECVEYVK SFNIPLLVLGGGGYTVRNVARCWTYETSLLVEEAIS 320 Macaca mulatta 241 NQVVDFYQPTCIVLQCGADSLGCDRLGCFNLSIRGHGECVEYVKSFNIPLLVLGGGGYTVRNVARCWTYETSLLVEEAIS 320 $\begin{array}{ll}\text { Bos taurus } & 241 \text { NQVVDFYQPTCIVLQCGAD SLGCDRLGCFNL SIRGHGECVEYVK SFNIPLLVLGGGGYTVRNVARCWTYET SLLVEEAIS } 320 \\ \text { Danio rerio } & 241 \text { KQVVDFYQPTCIVLQCGADSLGCDRLGCFNLSIRGHGECVEFVKGFKIPLLVLGGGGYTVNVARCWTFETSLLVEESIS } 320\end{array}$
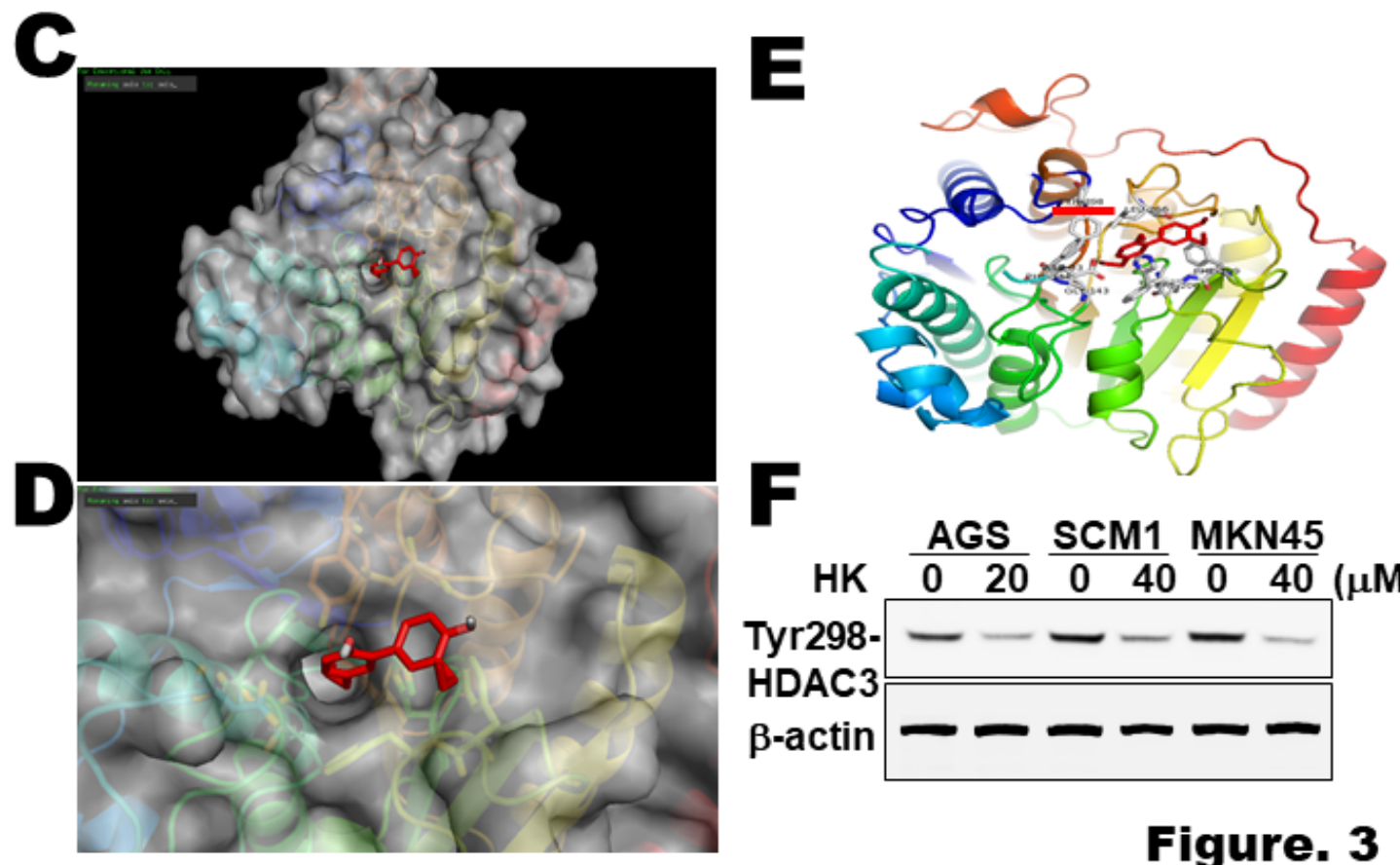

\section{AGS SCM1 MKN45}

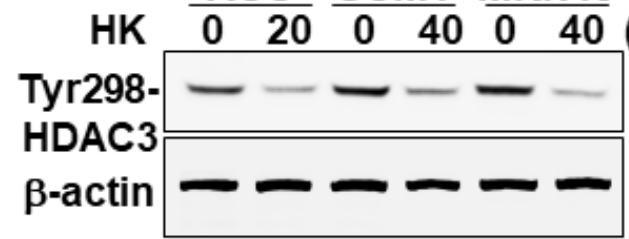

Figure. 3

\section{Figure 3}

Honokiol inhibited HDAC3 enzymatic activity in gastric cancer cells. The chemical structure of Honokiol revealed binding modes in HDAC3 by molecular docking simulation was also observed. General and local overviews of the best interaction after automated docking of binding Honokiol (red) to the active site of 
HDAC3. (A) Exposure to Honokiol inhibits HDAC3 enzymatic activity in gastric cancer cells AGS, SCM1 and MKN45 in a dose-dependent manner. Trichostatin A (TSA) served as a positive control. HDAC3 activity was measured by colorimetric HDAC3 Activity Assay Kit. The results are representative of at least six independent experiments and presented as mean $\pm S D$; ${ }^{*} p<0.01$ versus untreated cells (B) Alignment of the sequences of the HDAC3. Sequences are the amino acids for residues 241-320 of the proteins. Organisms were Homo sapiens, Homo sapiens, Mus musculus, Rattus norvegicus, Macaca mulatta, Bos Taurus, Danio rerio. Sequences are from Genebank. (C) Surface representation of the ligand-receptor binding. Honokiol and HDAC3 are shown in surface representation. (D) Local interaction positions of the HDAC active site. The cave entrance of zoom-in binding mode of HDAC3 and Honokiol are shown. (E) The molecular structure of human HDAC3 with Tyr 298 is depicted as ribbon diagram, with a-helices, $\beta$ pleated sheets, and loops. The binding mode of HDAC3 and Honokiol are shown targeting Tyr 298 representation. The 3D representation of the interactions of Honokiol binding to the HDAC3 active site, as generated by PyMol. (F) Honokiol-treated cancer cells (AGS $20 \mu \mathrm{M}$; SCM1 $40 \mu \mathrm{M}$; MKN45 $40 \mu \mathrm{M}$ ) markedly reduced the specific antibody targeting Tyr (Y298) of HDAC3 at 2 hours, whole cell lysates as evaluated by western blotting. The results are representative of at least six independent experiments and presented as mean $\pm S D$. 
A gegtaggggacaggcgg

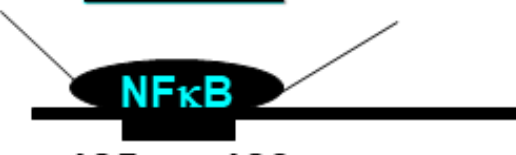

GTGCTTGCGCAGCACGCACGG

D

$-465 \sim-460$

NFKB

HDAC3



120

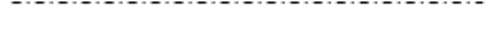

C/EBP $\beta$

100

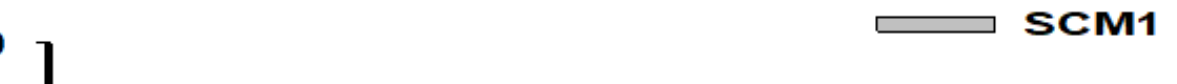

80

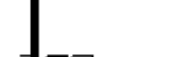

60

40

20

HK (UM̂)

$-21 \sim-14$

C/EBP $\beta$



AGS

SCM1

shCalp-2

shEGFP

C

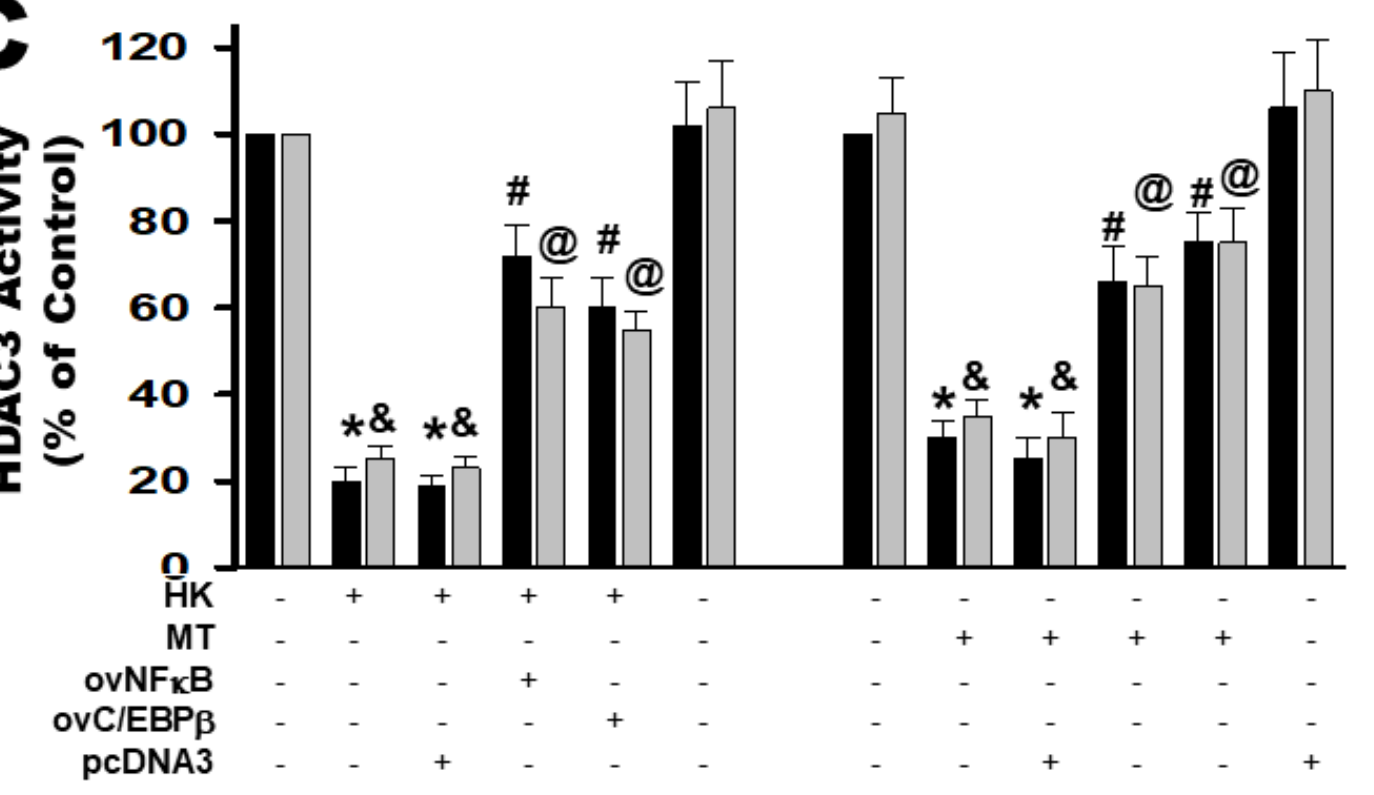

Figure. 4

Figure 4

Honokiol suppressed HDAC3-regulated NFKB and C/EBP $\beta$ signaling in gastric cancer cells. (A) The sequences of NFKB and C/EBP $\beta$ in HDAC3 promoter are presented, indicating the sub-fragments used in this study and the consensus minimal promoter (underlined as indicated). Promoter regulation in the HDAC3 promoter-flanking region (-465 -460) contained the cis-acting elements NFKB DNA binding site. HDAC3 promoter-flanking region (-21 -14) contained the transcription repression C/EBP $\beta$ DNA binding 
site. (B) Honokiol reduced the activation of C/EBPB (right) and NFKB (left). The relative activity was measured by dual luciferase activity kit as described in "Materials and Methods". All data are presented as mean $\pm S D$ of six separate determinations. \#@p<0.05; *\&p<0.01. (C) SCM1 or AGS gastric cancer cells were treated with of Honokiol $(40 \mu \mathrm{M})$ or melatonin $(\mathrm{MT} ; 100 \mathrm{mM})$ for 24 hours, and transiently transfected with NFKB plasmid or C/EBP $\beta$ plasmid for HDAC3 activity. This was determined in a test sample. All data are presented as mean \pm SD of six separate determinations. \#@p<0.05; *\&p<0.01.
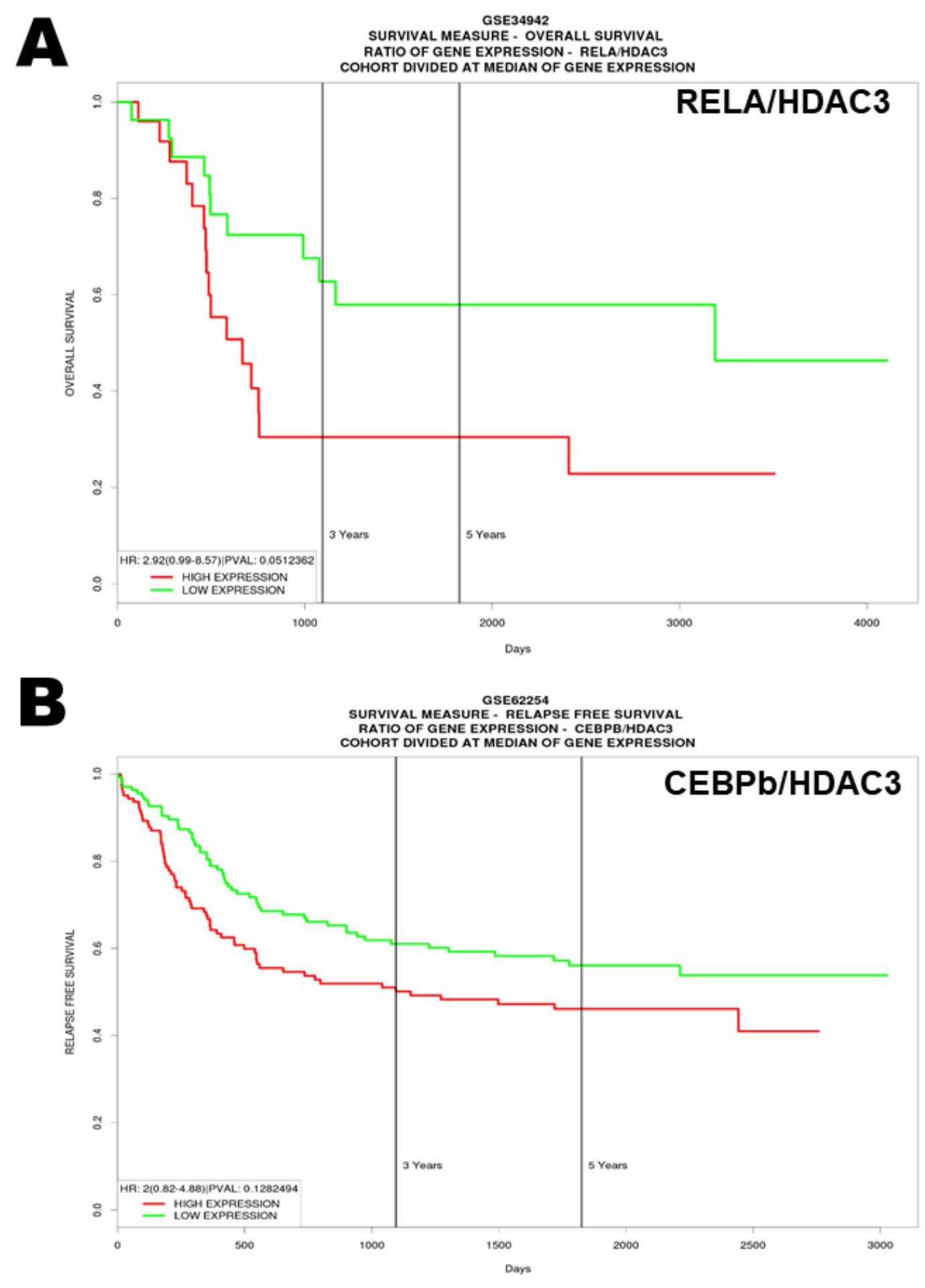

Figure. 5

Figure 5 
High levels of NFKB-p65 (RELA), C/EBP 3 , and HDAC3 expression were associated with decreased overall survival probability in gastric cancer. (A) Data were from the Survival dataset (GSE34942) through a comprehensive search using SurvExpress for RELA and HDAC3 evaluation. (B) Enhancements of the existing database (GSE62254) PROGgeneV2 for C/EBPß and HDAC3.

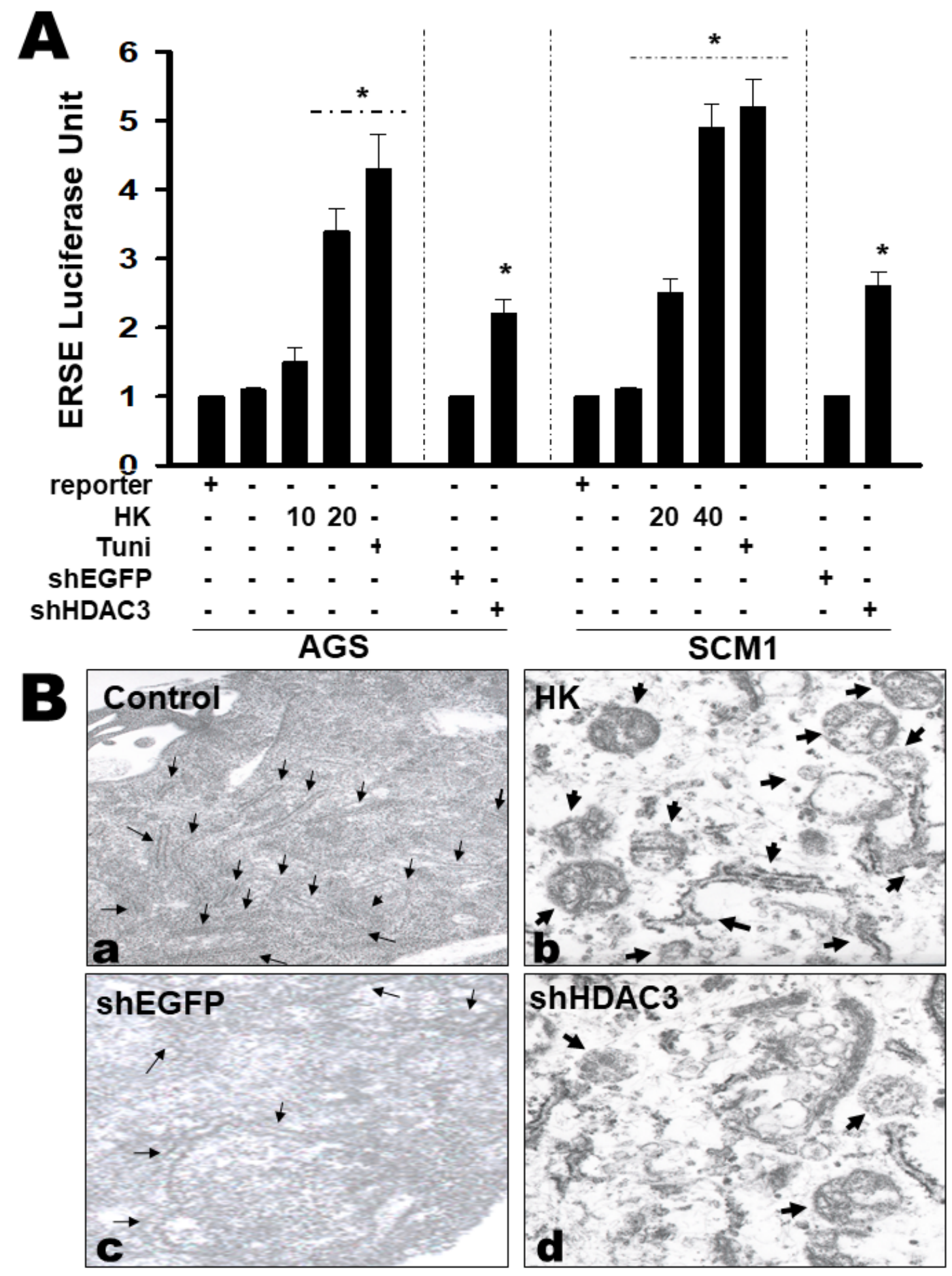

Figure. 6

Figure 6 
Honokiol and gene silencing of HDAC3 induced ER stress in gastric cancer cells. (A) The Cignal TCF/LEF reporter assay was designed to monitor the activity of Wnt signal transduction pathways in cultured AGS or SCM1 cells after transfection with shEGFP or shHDAC3 and then exposed to Honokiol after $18 \mathrm{hr}$. The data are representative of at least six independent experiments. Data are presented as mean $\pm S D(n=6)$. ERSE reporter contained inducible transcription-factor-responsive GFP reporter; Negative control included GFP reporter construct with GFP expression controlled by a minimal promoter; Positive control consist of constitutively expressing GFP construct. ER stress inducer Tunicamycin $0.1 \mu \mathrm{g} / \mathrm{ml}$ was the positive control. All data are presented as mean $\pm S D$ of six separate determinations. ${ }^{*} p<0.05$ (B) ER stress was examined by transmission electron microscopy. Cells were collected and visualized by electron microscopy as described in the "Materials and Methods". The results shown are representative of typical experiments. a) Control AGS cells; b) Honokiol-treated cell displays ER dilation; c) cells transfection shEGFP alone; and d) cells transfection shHDAC3 alone induces serious ER dilation with increased distention and a fragmented organelle. Arrows indicate dilated ER; Original magnifications, $9800 \mathrm{~K}$ 


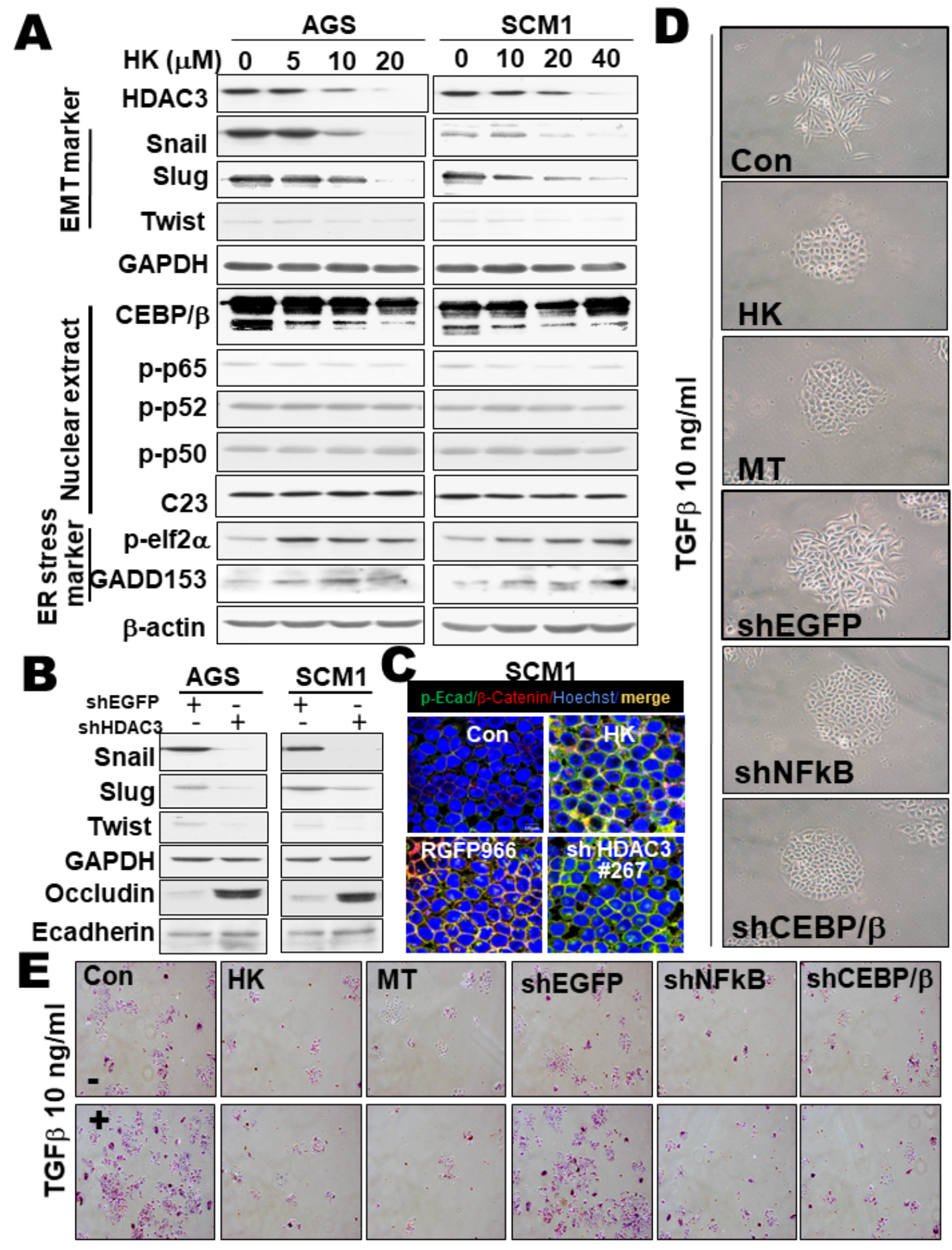

Figure. 7

Figure 7

Honokiol and gene silencing of HDAC3 attenuated the EMT markers, cell invasion, and sphere formation ability of gastric cancer cells. (A) Honokiol (HK) had a dose-dependent depend effect on cells, as detected by western blotting. The EMT markers (Snail, Slug, Twist), transcription factor (C/EBP $\beta$, NFKB p-p65, pp52 and p50), ER stress markers (p-elf2a, GADD153) were all evaluated. The images are representative of at least five independent experiments. (B) Knockdown HDAC3 by gene silencing of HDAC3 
(shHDAC3\#267) suppressed EMT markers and increased Occludin, but not Ecadherin or p-Ecadherin (data not shown). (C) Cells were exposed to Honokiol, HDAC3 inhibitor RGFP966, and gene silencing of HDAC3 (shHDAC3\#267) in a dose-dependent manner and then fixed and incubated with monoclonal antibodies against specific p-Ecadherin (green), $\beta$-Catenin (red) and Hoechst (blue). Confocal images for p-Ecadherin, $\beta$-Catenin, and Hoechst were examined and quantified as described in the "Materials and Methods". The images are representative of at least five independent experiments. The average intensity per cell is presented as mean $\pm S D$. (D) SCM1 cells were treated with $T G F \beta(10 \mathrm{ng} / \mathrm{mL})$ in the presence or absence of Honokiol (HK), melatonin (MT), or transfection with expressing shEGFP (control), shNFKB, or shCEBPB and subsequently seeded onto 24 well plates for one week for morphological examination. The images are representative of at least five independent experiments. (E) For invasion assay, SCM1 cells were treated with TGF $\beta(10 \mathrm{ng} / \mathrm{mL})$ in the presence or absence of Honokiol (HK), melatonin (MT), or transfection with expressing shEGFP (control), shNFKB, or shCEBPB. Cells were loaded onto the upper compartments of Matrigel $(30 \mu \mathrm{g} / \mathrm{mL})$-coated plates and incubated for 16 hours. After fixing and staining with $0.6 \%$ hematoxylin and $0.5 \%$ eosin, the number of migrated or invasive cells was counted. All of the images are representative of at least five independent experiments.

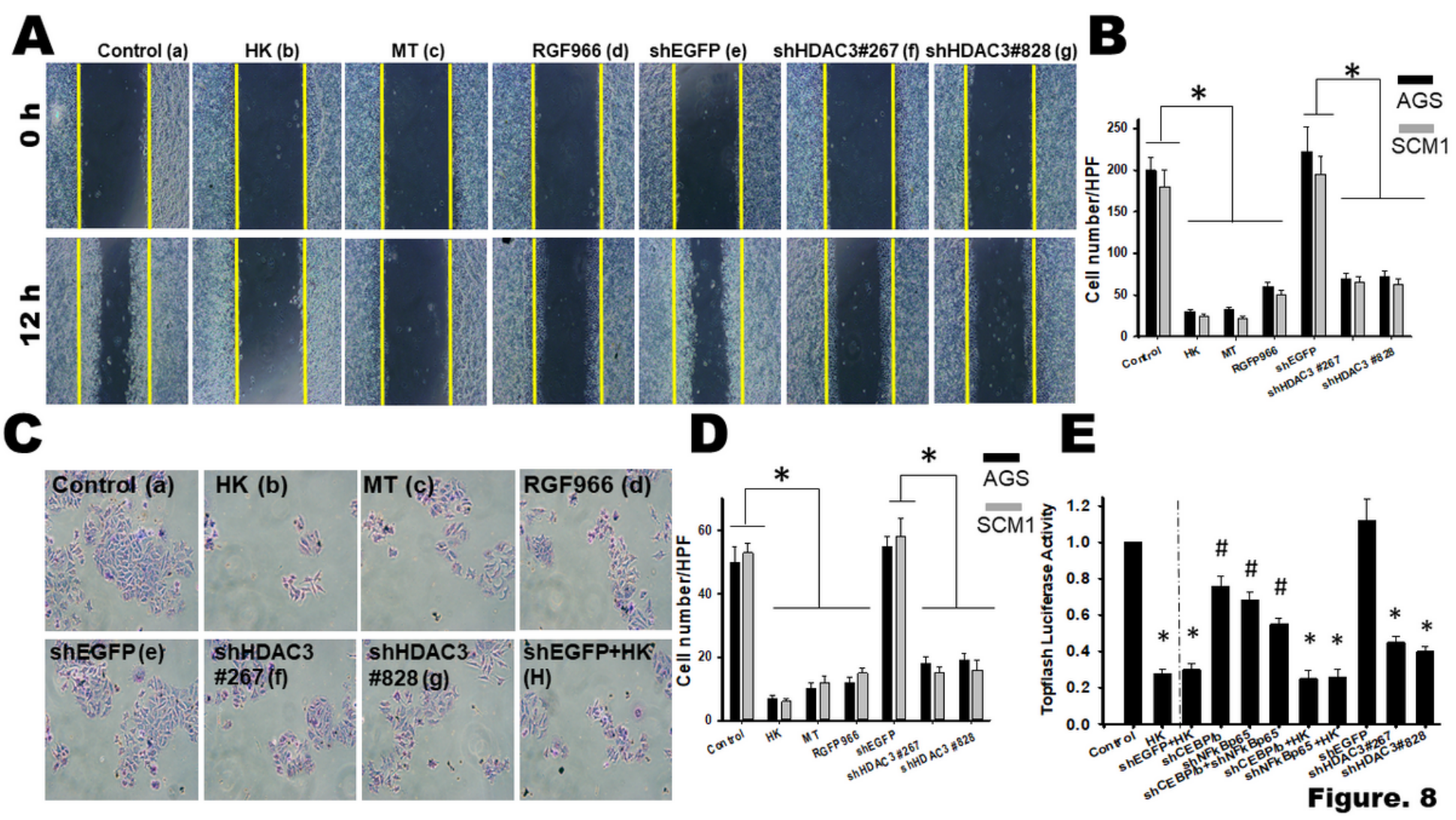

\section{Figure 8}

Suppression of HDAC3 by Honokiol inhibited cell migration and invasion in gastric cancer cells. (A) Wound healing cell migration assays performed on monolayer cells revealed that Honokiol (HK), melatonin (MT), shHDAC3, and pharmacologic inhibitors RGFP966 mediated the inhibition of cellular migration in SCM1 cells. The graph shows the results of wound closure 18 hours after wounding, as derived from six independent experiments. Melatonin was the positive control. (B) Quantifications of 
numbers of migration in different treatments (counts/field) were calculated. All data are presented as mean $\pm S D(n=6) .{ }^{*} p<0.05$ compared to control. (C) For the cancer cell invasion assay, the numbers of migrated or invasive cells were counted after treatment. (D) Quantification of numbers of invasions in different treatments (counts/field) was made. All data are presented as mean $\pm S D(n=6) .{ }^{*} p<0.05$ compared with control. Cancer cells were co-transfected with TOPFlash or FOPFlash luciferase reporter genes along with renilla luciferase. After 6 hours, cells were left untreated (no treatment) or treated with Honokiol (HK) or as indicated silencing gene. After an additional 36 hours, cells were harvested and luciferase levels were determined; firefly luciferase was normalized to renilla. TOPFlash activity was highly induced in all six populations of cells and this was inhibited by Honokiol. As negative control, FOPFlash showed minimal response to Honokiol. TOPFlash inhibition by Honokiol was more robust in the SCM1 than in the AGS cell line. The experiment was done five with similar results. $\# p<0.05 ;{ }^{*}<0.001$.
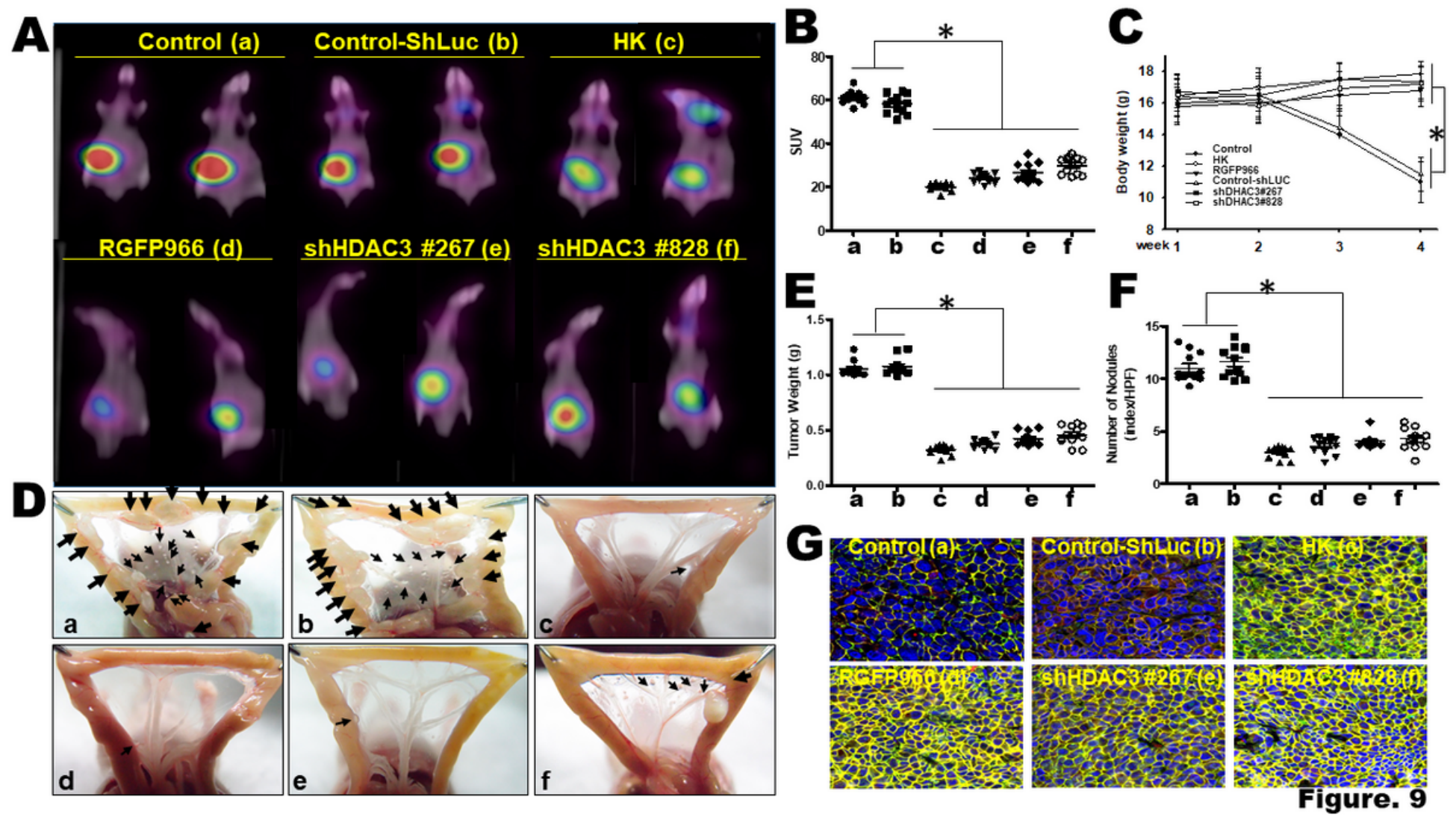

\section{Figure 9}

Treatments with Honokiol, HDAC3 gene silencing, or RGFP966 inhibited the metastasis of gastric tumors in a xenograft tumor mouse model. Mice received intra-peritoneal implants of MKN45 cells and treated with Honokiol (5 mg/kg, twice/weekly, intra-peritoneal injection), RGFP966 (5 mg/kg, twice/weekly, intraperitoneal injection) or knockdown HDAC3 expression (shHDAC3 \#267; \#828). (A) Randomized grouping of Honokiol-treated mice. Tumor burden was measured twice per week using PET/CT imaging. A representative image shows peritoneal dissemination of animals. The results are representative of at least 8-12 independent experiments. (B) Quantifications of representative images showing peritoneal dissemination of therapy-treated animals and controls. All data are presented as mean $\pm S D$. $n=8-12$ ). ${ }^{*} p<0.05$ compared to controls $(C)$ The body weight of all groups of mice was presented as a bar graph. 
All data are presented as mean $\pm S D(n=8-12) .{ }^{*} p<0.05$ compared to controls (D) Photomacrographs of metastatic peritoneal nodules vision of animals inoculated with MKN45 gastric cancer cells with or without scramble, knockdown gene treatment (arrows shown). The maximum intensity image of nude mice (tumor control and scramble) showed many metastatic nodules in the mesentery of the control mice inoculated with MKN45 cells. In contrast, peritoneal metastatic nodules were observed sporadically in Honokiol treated or gene silencing shHDAC3 or RGFP966-treated or mice. (E) The weights of metastasis mesenteric tumors presented as a bar graph. (F) Quantifications of nodule number. All data are presented as mean $\pm S D(n=8-12)$. ${ }^{\star} p<0.05$ compared to controls $(G)$ Animal tissues staining for Honokiol, HDAC3 inhibitor RGFP966, and gene silencing of HDAC3 expression (shHDAC3 \#267; \#828) probed with monoclonal antibodies against specific p-Ecadherin (green), $\beta$-Catenin (red), and Hoechst (blue). Confocal images for $p$-Ecadherin, $\beta$-Catenin, and Hoechst were examined and quantified as described in the "Materials and Methods". The images are representative of at least six independent experiments. The average intensity per cell is presented as mean \pm SD.

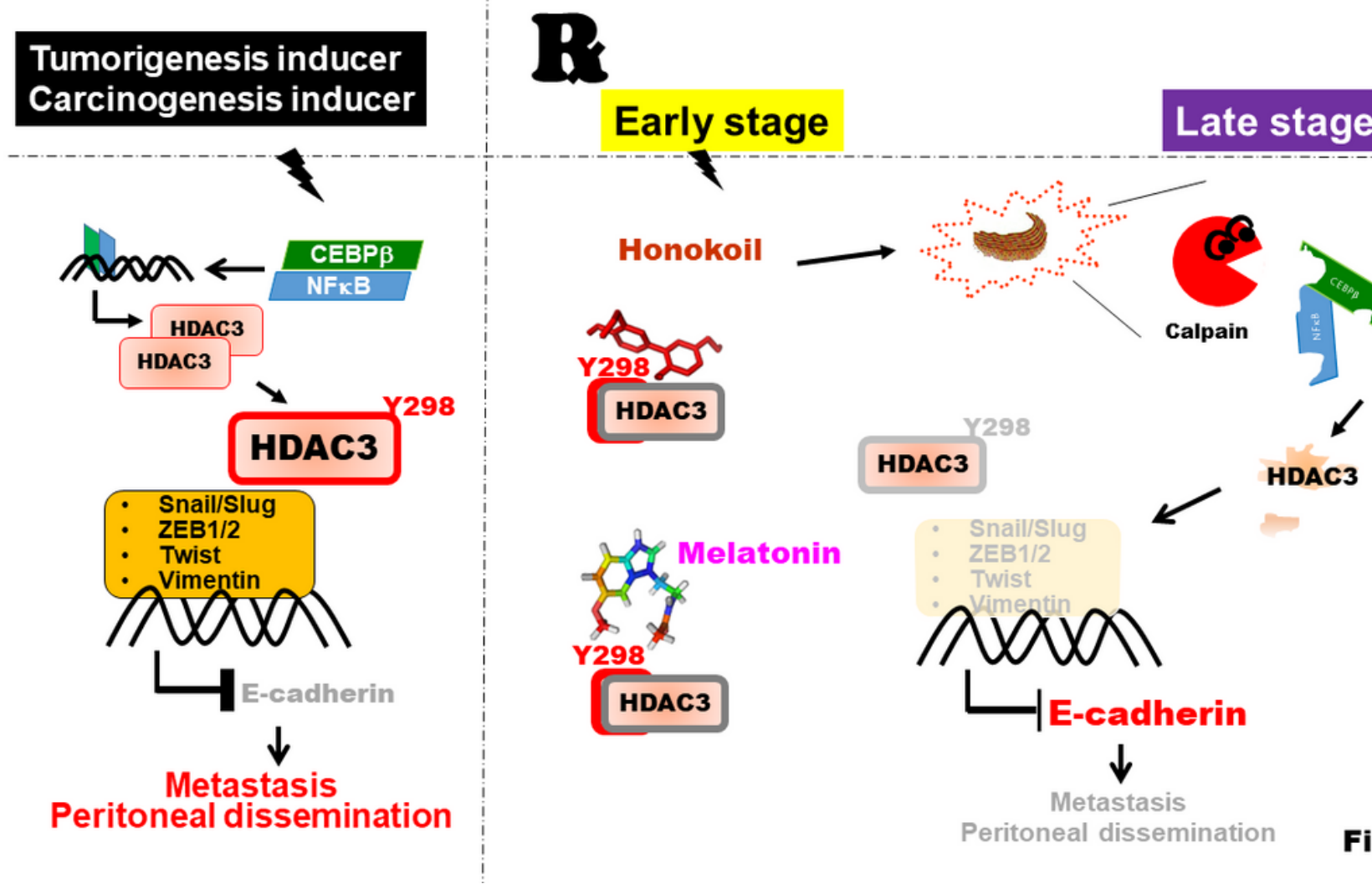

Figure. 10

Figure 10

Conceptual model showing the working hypothesis on the interaction among Honokiol, HDAC3, and ER stress in the peritoneal dissemination of gastric cancer. In the schematic of the proposed mechanism for the role of Honokiol targeting HDAC3 by ER stress cascade and mitigating the peritoneal spread of gastric cancer. Honokiol-induced ER stress activated calpain activity targeted HDAC3 and blocked of Tyr298 phosphorylation, subsequently blocked cooperating with EMT transcription factors and cancer progression. The present study provides evidence to demonstrate that HDAC3 is a positive regulator of EMT and metastatic growth of gastric cancer cells. The findings here imply that overexpressed HDAC3 is 
a potential therapeutic target for honokiol to reverse EMT and prevent gastric cancer migration, invasion, and metastatic dissemination.

\section{Supplementary Files}

This is a list of supplementary files associated with this preprint. Click to download.

- SupplementaryFig1.tif

- SupplementaryFig2.tif

- SupplementaryFig3.tif

- SupplementaryFig4.tif

- SupplementaryFig5.tif

- SupplementaryTable1.tif

- SupplementaryTable2.tif 